See discussions, stats, and author profiles for this publication at: https://www.researchgate.net/publication/338388237

Positivity-preserving finite volume scheme for compressible two-phase flows in anisotropic porous media : the densities are depending on the physical pressures

Article in Journal of Computational Physics · January 2020

DOI: 10.1016/j.jpp.2020.109233

3 authors:

Mustapha Ghilani

Université Moulay Ismail

16 PUBLICATIONS 133 CITATIONS

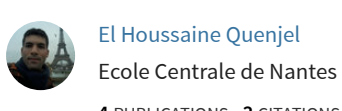

SEE PROFILE

SEE PROFILE

P. Mazen Saad

Ecole Centrale de Nantes

74 PUBLICATIONS 825 CitATIONS

SEE PROFILE

Some of the authors of this publication are also working on these related projects:

roject Approximation of two-phase flow View project 


\title{
Positivity-preserving finite volume scheme for compressible two-phase flows in anisotropic porous media : the densities are depending on the physical pressures
}

\author{
Mustapha GHILANI ${ }^{1}$, El Houssaine QUENJEL ${ }^{2}$, and Mazen SAAD ${ }^{3}$ \\ ${ }^{1}$ Moulay Ismaïl University, ENSAM B.P. 15290 EL Mansour 50500 Meknès, Morocco. \\ m.ghilani@ensam-umi.ac.ma \\ ${ }^{1}$ University of Nice Sophia-Antipolis, LJAD, CNRS UMR 7351, and COFFEE team, INRIA Sophia \\ Antipolis Méediterranée, Parc Valrose 06108 Nice Cedex 02, France. quenjel@unice.fr \\ ${ }^{3}$ Ecole Centrale de Nantes, LMJL, CNRS UMR 6629, 1 rue de la Nöé, 44321 Nantes, France. \\ mazen.saad@ec-nantes.fr
}

January 15, 2020

\begin{abstract}
We are concerned with the approximation of solutions to a compressible two-phase flow model in porous media thanks to an enhanced control volume finite element discretization. The originality of the methodology consists in treating the case where the densities are depending on their own pressures without any major restriction neither on the permeability tensor nor on the mesh. Contrary to the ideas of [23], the point of the current scheme relies on a phaseby-phase "sub"-unpwinding approach so that we can recover the coercivity-like property. It allows on a second place for the preservation of the physical bounds on the discrete saturation. The convergence of the numerical scheme is therefore performed using classical compactness arguments. Numerical experiments are presented to exhibit the efficiency and illustrate the qualitative behavior of the implemented method.
\end{abstract}

\section{Statement of the continuous model}

The displacement of fluids in porous media is a subject of significant importance among many fields of engineering and industries [5, 11, 24]. Typical applications include enhanced oil recovery, nuclear waste management and soil remediation.

Under some realistic conditions, compressible two-phase flows in porous media are often modeled by the mass conservation equation for each phase $\alpha$ where the velocity is expressed by the generalized Darcy-Muscat law. We here consider a gaseous phase indexed by $g$ and a liquid fluid denoted by $w$. The porous medium $\Omega$ is assimilated to a polygonal connected bounded open domain of $\mathbb{R}^{d}$. Let $t_{f}$ be in $(0,+\infty)$. For each phase $\alpha \in\{g, w\}$, the continuity equation states $[11]$ :

$$
\phi \partial_{t}\left(\rho_{\alpha} s_{\alpha}\right)+\nabla \cdot\left(\rho_{\alpha} \mathbf{V}_{\alpha}\right)+\rho_{\alpha} q^{\alpha}=0 \quad \text { in } Q_{t_{f}}:=\Omega \times\left(0, t_{f}\right),
$$

where $\phi$ is the porosity of the medium and $\rho_{\alpha}\left(p_{\alpha}\right), s_{\alpha}, \mathbf{V}_{\alpha}, q^{\alpha}$ are respectively the density, the saturation, the flow speed and the source term of the fluid $\alpha$. The expression of the velocity $\mathbf{V}_{\alpha}$ 


$$
\mathbf{V}_{\alpha}=-\frac{K_{r \alpha}}{\mu_{\alpha}} \Lambda\left(\nabla p_{\alpha}-\rho_{\alpha} \overrightarrow{\mathbf{g}}\right)
$$

where $K_{r \alpha}$ stands for the relative permeability, $\mu_{\alpha}$ is the dynamic viscosity, and $p_{\alpha}$ is the pressure of the phase $\alpha$. The matrix $\Lambda$ designates the intrinsic permeability of the medium. The vector $\vec{g}$ accounts for the gravitational acceleration. The ratio $K_{r \alpha} / \mu_{\alpha}$ is usually termed as the $\alpha$-phase mobility. We assume that domain of study is completely filled by the two fluids, which means that

$$
s_{g}+s_{w}=1 .
$$

The relationship between the nonwetting phase and the wetting one is given by the capillary pressure law :

$$
p_{c}\left(s_{g}\right)=p_{g}-p_{w} .
$$

Notice that this function and the mobilities $M_{\alpha}$ are strongly nonlinear and they are only defined in terms of the saturations. We point out that $M_{\alpha}\left(s_{\alpha}=0\right)=0$. This is referred to as the degeneracy issue. It is encountered when the phase $\alpha$ disappears somewhere within $\Omega$.

Following [27], the volumetric source-sink rate $q^{\alpha}$ is set to $q^{\alpha}=s_{\alpha} f^{P}-s_{\alpha}^{I} f^{I}$, where the functions $f^{I}, f^{P}$ measures somehow the injection and the production rates of the involved fluids. The coefficient $s_{\alpha}^{I}$ is a known $\alpha$-phase saturation which corresponds to the ratio of the injected fluid within the medium of interest.

We hereafter choose both pressure $p_{g}$ and $p_{w}$ as the primary unknowns. Besides to equations (1.1)-(1.4), we prescribe the boundary conditions and the initial data as follows :

$$
\begin{cases}\rho_{\alpha} \mathbf{V}_{n} \cdot \mathbf{n}=\rho_{\alpha} \mathbf{V}_{w} \cdot \mathbf{n}=0 & \text { on } \Gamma_{N} \times\left(0, t_{f}\right) \\ p_{g}=p_{g}^{D} \text { and } p_{w}=p_{w}^{D} & \text { on } \Gamma_{D} \times\left(0, t_{f}\right) \\ p_{g}(\cdot, 0)=p_{g}^{0} \text { and } p_{w}(\cdot, 0)=p_{w}^{0} & \text { in } \Omega\end{cases}
$$

where $\left\{\Gamma_{N}, \Gamma_{D}\right\}$ is an appropriate partition of the boundary $\partial \Omega=\Gamma_{N} \cup \Gamma_{D}$ with $\left|\Gamma_{D}\right|>0$ and $\mathbf{n}$ is the outward unit normal to $\Gamma_{N}$. For the sake of simplicity, one may take $p_{g}^{D}=p_{w}^{D}=0$ in the analysis of the numerical scheme.

Even if it is not involved in the governing equations of compressible flows, the concept of the global pressure [11] is of great help for the analysis of both the continuous and the discrete system. Its introduction is necessary to handle the degeneracy issue of the hyperbolic terms since the gradient $\nabla p_{\alpha}$ is lost whenever $s_{\alpha}=0$.

In the sequel, $p$ denotes the global pressure. We also consider $M\left(s_{g}\right)=M_{w}\left(1-s_{g}\right)+M_{g}\left(s_{g}\right)$ as the total mobility. We then recall that $p$ is defined via the following relationship

$$
M\left(s_{g}\right) \nabla p=M_{w}\left(s_{w}\right) \nabla p_{w}+M_{g}\left(s_{g}\right) \nabla p_{g} .
$$

The function $p$ is an intermediary pressure between the gas pressure and that of the wetting phase such that

$$
p=p_{g}-\widehat{p}_{g}\left(s_{g}\right)=p_{w}+\widehat{p}_{w}\left(s_{g}\right),
$$

where the artificial pressures $\widehat{p}_{g}, \widehat{p}_{w}$ read

$$
\widehat{p}_{g}\left(s_{g}\right)=\int_{0}^{s_{g}} \frac{M_{w}(1-u)}{M(u)} p_{c}^{\prime}(u) \mathrm{d} u \text { and } \widehat{p}_{w}\left(s_{g}\right)=\int_{0}^{s_{g}} \frac{M_{g}(u)}{M(u)} p_{c}^{\prime}(u) \mathrm{d} u .
$$

We next define the capillary term $\mathcal{B}$ :

$$
\mathcal{B}\left(s_{g}\right)=\int_{0}^{s_{g}} \frac{M_{w}(1-u) M_{g}(u)}{M(u)} p_{c}^{\prime}(u) \mathrm{d} u .
$$

In light of the above expressions, one formally has

$$
M_{w}\left(s_{w}\right) \nabla p_{w}=M_{w}\left(s_{w}\right) \nabla p+\nabla \mathcal{B}\left(s_{g}\right), \quad M_{g}\left(s_{g}\right) \nabla p_{g}=M_{g}\left(s_{g}\right) \nabla p-\nabla \mathcal{B}\left(s_{g}\right) .
$$


Whence, the following equality holds

$$
M_{g}\left(s_{g}\right)\left|\nabla p_{g}\right|^{2}+M_{w}\left(s_{w}\right)\left|\nabla p_{w}\right|^{2}=M\left(s_{g}\right)|\nabla p|^{2}+\frac{M_{w}\left(1-s_{g}\right) M_{g}\left(s_{g}\right)}{M\left(s_{g}\right)}\left|\nabla p_{c}\left(s_{g}\right)\right|^{2} .
$$

At the discrete level [32], this identity is destroyed. It can however be substituted by three inequalities whose proofs are not evident. To simplify these proofs, we rather propose to use the features of the function $\xi$, instead of $\mathcal{B}$, given by

$$
\xi\left(s_{g}\right)=\int_{0}^{s_{g}} \frac{\sqrt{M_{w}(1-u) M_{g}(u)}}{M(u)} p_{c}^{\prime}(u) \mathrm{d} u .
$$

According to (1.10), one gets

$$
M_{g}\left(s_{g}\right)\left|\nabla p_{g}\right|^{2}+M_{w}\left(s_{w}\right)\left|\nabla p_{w}\right|^{2}=M\left(s_{g}\right)|\nabla p|^{2}+M\left(s_{g}\right)\left|\nabla \xi\left(s_{g}\right)\right|^{2} .
$$

Based on (1.11), a discrete inequality will be established later in Section 4 using similar ideas as provided in $[29,32]$. As a consequence, the discrete energy of the whole system is controlled by estimating the approximate gradient of the global pressure and the capillary potential regardless the phase disappearance issue. We would like to emphasize that the main strengths of the underlined inequality consists in omitting the heavy calculus and improving the convergence proofs already carried out in $[32,33]$.

Now, in order to define in a proper sense the weak solutions to the system (1.1)-(1.5) we need to specify some relevant assumptions on the physical data. These hypotheses are standard in the compressible two-phase flow context.

$\left(\mathbf{A}_{0}\right)$ The initial pressures $p_{g}^{0}, p_{w}^{0}$ are $L^{2}(\Omega)$-functions so that the $\alpha$-phase initial saturation $s_{\alpha}^{0}$ belongs to $L^{\infty}(\Omega)$ with $0 \leq s_{\alpha}^{0}(x) \leq 1$ a.e. $x \in \Omega$.

$\left(\mathbf{A}_{1}\right)$ The porosity $\phi$ is in $L^{\infty}(\Omega)$ such that there exist $\phi_{0}, \phi_{1}>0: \phi_{0} \leq \phi(x) \leq \phi_{1}$ a.e. $x \in \Omega$.

$\left(\mathbf{A}_{2}\right)$ The mobility $M_{\alpha}$ of the phase $\alpha$, is an increasing continuous function from $[0,1]$ to $\mathbb{R}$ where we take into account the extension : $M_{\alpha}\left(s_{\alpha}\right)=0$ for every $\left.\left.s_{\alpha} \in\right]-\infty, 0\right]$. Moreover, the total mobility is bounded far away from 0 i.e. there exists $m_{0}>0$ such that

$$
m_{0} \leq M\left(s_{g}\right)=M_{g}\left(s_{g}\right)+M_{w}\left(1-s_{g}\right), \quad \forall s_{g} \in[0,1] .
$$

$\left(\mathbf{A}_{3}\right)$ The intrinsic permeability $\Lambda$ is a positive-definite matrix and essentially bounded. It is further uniformly elliptic i.e. there exist positive constants $\underline{\Lambda}$ and $\bar{\Lambda}$ such that

$$
\underline{\Lambda}|\zeta|^{2} \leq \Lambda(x) \zeta \cdot \zeta \leq \bar{\Lambda}|\zeta|^{2} \text {, for all } \zeta \in \mathbb{R}^{d} \text { and a.e. } x \in \Omega .
$$

$\left(\mathbf{A}_{4}\right)$ The injection and the production terms $f^{I}, f^{P}$ are two nonnegative functions of $L^{2}\left(Q_{t_{f}}\right)$. In addition, for $\alpha=g, w, s_{\alpha}^{I}$ is a bounded function such that $s_{g}^{I}+s_{w}^{I}=1$ a.e. $(x, t) \in Q_{t_{f}}$.

$\left(\mathbf{A}_{5}\right)$ The density $\rho_{\alpha} \in \mathcal{C}^{1}(\mathbb{R}, \mathbb{R})$ is increasing and uniformly bounded : $0<\rho_{0} \leq \rho\left(p_{g}\right) \leq \rho_{1}$, for some positive constants $\rho_{0}, \rho_{1}$.

$\left(\mathbf{A}_{6}\right)$ The capillary pressure function $p_{c}\left(s_{g}\right) \in \mathcal{C}^{1}\left([0,1], \mathbb{R}^{+}\right)$is increasing. We assume that $p_{c}(0)=0$.

$\left(\mathbf{A}_{7}\right)$ The function $\xi^{-1}$ is $\theta$-Hölder on $[0, \xi(1)]$ with $\theta \in(0,1)$. This means that there exists a positive constant $L_{\xi}$ such that for all $a, b \in[0, \xi(1)],\left|\xi^{-1}(a)-\xi^{-1}(b)\right| \leq L_{\xi}|a-b|^{\theta}$.

Remark 1.1. The requirement $p_{c}(0)=0$ in Assumption $\left(\boldsymbol{A}_{6}\right)$ stands for a compatibility condition. Indeed, using (1.7) and (1.8) we find

$$
\widehat{p}_{g}\left(s_{g}\right)+\widehat{p}_{w}\left(s_{g}\right)=p_{c}\left(s_{g}\right)=p_{g}-p_{w} .
$$

In addition, the boundary constraint $p_{g}^{D}=p_{w}^{D}=0$ implies $s_{g}^{D}=0$. As a consequence, the global pressure vanishes on $\Gamma_{D}$ i.e. $p^{D}=0$. 
We consider the classical Sobolev space

$$
H_{\Gamma_{D}}^{1}(\Omega)=\left\{u \in H^{1}(\Omega) / u=0 \text { on } \Gamma_{D}\right\}
$$

We recall that it is a Hilbert space endowed with the norm

$$
\|u\|_{H_{\Gamma_{D}}^{1}(\Omega)}=\|\nabla u\|_{L^{2}(\Omega)^{d}} .
$$

Definition 1.1. (Weak solutions) Under Assumptions $\left(\boldsymbol{A}_{0}\right)-\left(\boldsymbol{A}_{7}\right)$, we call a weak solution to the continuous model (1.1)-(1.5) any couple of measurable functions $\left(p_{g}, p_{w}\right)$ verifying for every $\alpha \in\{g, w\}$

$$
\begin{gathered}
s_{g}=p_{c}^{-1}\left(p_{g}-p_{w}\right), \quad s_{w}=1-s_{g}, \text { a.e. in } Q_{t_{f}} \\
0 \leq s_{\alpha} \leq 1 \text { a.e. in } Q_{t_{f}}, \xi\left(s_{g}\right) \in L^{2}\left(0, t_{f} ; H_{\Gamma_{D}}^{1}(\Omega)\right), \\
p_{\alpha} \in L^{2}\left(0, t_{f} ; L^{2}(\Omega)\right), \sqrt{M_{\alpha}\left(s_{\alpha}\right)} \nabla p_{\alpha} \in L^{2}\left(0, t_{f} ; L^{2}(\Omega)^{d}\right)
\end{gathered}
$$

and for every $\psi \in \mathcal{C}_{c}^{\infty}\left(\bar{\Omega} \times\left[0, t_{f}\right)\right)$, with $\psi=0$ on $\Gamma_{D} \times\left[0, t_{f}\right)$, there holds

$$
\begin{gathered}
-\int_{Q_{t_{f}}} \phi \rho_{\alpha}\left(p_{\alpha}\right) s_{\alpha} \partial_{t} \psi \mathrm{d} x \mathrm{~d} t-\int_{\Omega} \phi \rho_{\alpha}\left(p_{\alpha}^{0}\right) s_{\alpha}^{0} \psi(x, 0) \mathrm{d} x+\int_{Q_{t_{f}}} \rho_{\alpha}\left(p_{\alpha}\right) M_{\alpha}\left(s_{\alpha}\right) \Lambda \nabla p_{\alpha} \cdot \nabla \psi \mathrm{d} x \mathrm{~d} t \\
-\int_{Q_{t_{f}}} \rho_{\alpha}^{2}\left(p_{\alpha}\right) M_{\alpha}\left(s_{\alpha}\right) \Lambda \overrightarrow{\boldsymbol{g}} \cdot \nabla \psi \mathrm{d} x \mathrm{~d} t+\int_{Q_{t_{f}}} \rho_{\alpha}\left(p_{\alpha}\right) s_{\alpha} f^{P} \psi \mathrm{d} x \mathrm{~d} t=\int_{Q_{t_{f}}} \rho_{\alpha}\left(p_{\alpha}\right) s_{\alpha}^{I} f^{I} \psi \mathrm{d} x \mathrm{~d} t .
\end{gathered}
$$

We indicate that the mathematical model (1.1)-(1.5) admits a weak solution in the sense of Definition 1.1. This statement was the object of the work [27]. The key idea is to pass through artificial unknowns namely the global pressure in order to get a priori estimates on the system energy using of course the fundamental relationship (1.10). A simplified version of the same model is analyzed in [22] where the fluid densities are only functions in terms of the global pressure. Owing to the latter concept and extended techniques, an equivalent formulation for an immiscible compressible two-phase flow in porous media is proposed and studied in [2].

From a numerical point of view, many models of type (1.1)-(1.5) have been extensively examined. For instance, we refer to the relevant non-exhaustive list on the finite element schemes $[11,12,26,31,34]$. Discontinuous Galerkin methods for incompressible two-phase flows are given in $[15,30]$. In the last past years, plenty of computational approaches $[13,24]$ (the references are therein) have been widely implemented for solving numerically the underlined system. However, these methodologies lack of a rigorous mathematical analysis, namely the convergence of the numerical scheme. Thus, such an application to complex problems may lead to unforeseen issues that might consist, for instance, of nonphysical oscillations and loss of convergence. On the other hand, assuming restrictive assumptions either on the data or on the involved meshes, a few papers were dedicated to the convergence analysis of the numerical method for immiscible two-phase flow systems. For example, the pioneer two-point flux approximation schemes have been implemented and investigated in the documents [6, 29, 32]. Based on a phase-by-phase upstream technique, a finite volume and nonconforming finite element approach was suggested in [33] for the same model. We stress that the discretizations mentioned in the last references rest essentially on the nonnegativity of transmissibility coefficients. A similar constraint was also imposed in $[1,18]$ for conducting the proof of the convergence results. This latter condition is too stringent and it holds on particular data and meshes [14]. To deal with the anisotropic model with a slight compressibility factor, we recently investigated a control volume finite element method in [23]. This approach was performed using the global pressure formulation for the system of interest where the gas density depends on the global pressure. The point is to view the elliptic part like a hyperbolic one as done in $[8,10]$.

In the present article, the system is composed of two degenerate parabolic equations. The diffusion behavior of the model is implicitly included in the convection terms. This is due 
of course to the capillary effects. From a numerical perspective, when some transmissibility coefficients are nonpositive the coercivity on the discrete gradients is lost. The same issue has been reported for instance in $[14,19]$ for many schemes. Particularly, the control volume finite element scheme proposed in [23], the references are therein, can provide negative stiffness coefficients when the permeability tensor is anisotropic or the simplicial mesh does not verify the Delaunay condition [16]. In addition, the latter scheme is based on a classical upstreaming technique which can not be applied to the present model since the system of interest is nonlinear, degenerate and its variables are strongly coupled. Then, the goal of our contribution is to develop and analyze a vertex-centered finite volume discretization so that we can handle in a proper way the coupling and the degeneracy issues. The particularity of our strategy hinges on a "sub-upwinding" scheme for the approximation of the connective fluxes. The objective is to recover the fundamental points consisting of both the discrete maximum principle and uniform estimates on the discrete gradient of the global pressure and on a new capillary term. These stability results are mandatory for the convergence analysis of the method, especially for establishing compactness properties. By the discrete maximum principle, we mean that the approximate saturations are always bounded between 0 and 1 . This fact is validated numerically on a sequence of triangular meshes for several anisotropies of the permeability matrix.

The rest of this paper is structured as follows. Section 2 surveys the discrete framework consisting of the spacial and temporal meshes, the discrete functional spaces and some related notations. Section 3 introduces the proposed numerical scheme. Section 4 is dedicated to the exposition and proofs of the pillared stability estimates of this paper. Section 5 is devoted to the convergence proof of the numerical scheme as well as the identification of the limits. Section 6 presents a series of numerical experiments. The objective is first to show the efficiency and the robustness of our approach on an academic example. Such a method is then illustrated on the considered compressible two-phase flow for different types of anisotropy.

\section{$2 \quad$ Discrete setting}

We here describe the used meshes as well as some relevant notations.

\subsection{Spatial discretizations of the porous medium}

In the framework of the vertex-centered finite volume methods we need to define at least two different kinds of meshes. In our case, the first one is a finite element partition of the domain $\Omega$ referred to as the primal mesh, on which the second one is built and it is termed as the dual mesh.

For the sake of simplicity we consider the two-dimensional case $d=2$. Let $\mathcal{T}_{h}$ be a conforming simplicial mesh of $\Omega$ in the sense of the finite element method [16]. Then, the intersection of two elements is either a vertex, an edge or the empty set. Let us denotes by $\mathcal{V}$ the set of the vertices of the mesh. This family is split into two subsets $\mathcal{V}_{D}$ and $\mathcal{V}_{D}^{c}$. In fact, $\mathcal{V}_{D}$ holds the vertices located on the boundary $\Gamma_{D}$ while $\mathcal{V}_{D}^{c}=\mathcal{V} \backslash \mathcal{V}_{D}$. The set $\mathcal{V}_{T}$ denotes the nodes of an element $T \in \mathcal{T}_{h}$. We define $x_{T}$ the barycenter, $h_{T}=\operatorname{diam}(T)$, and $|T|$ the Lebesgue measure of the triangle $T \in \mathcal{T}_{h}$. We designate by $r_{T}$ the diameter of the largest ball included in $T$. The size of the mesh $h$ is given by the maximum of the finite family $\left\{h_{T}, T \in \mathcal{T}_{h}\right\}$. As it is standard in the finite element literature, the triangulation in question should be regular in the sense that :

$$
\exists \Theta>0: \max _{T \in \mathcal{T}_{h}} \frac{h_{T}}{r_{T}} \leq \Theta, \quad \forall h \in\left(0, h_{0}\right) .
$$

As highlighted above, the dual mesh $\mathcal{A}_{h}$ is constructed on the primal mesh $\mathcal{T}_{h}$ and centered on its vertices. The elements of $\mathcal{A}_{h}$ are usually called control volumes. The set of triangles sharing $K \in \mathcal{V}$ is designated by $\mathcal{K}_{\mathcal{T}_{h}}$. In each triangle we consider the set $\mathcal{V}_{K T}:=\mathcal{V}_{T} \backslash\{K\}$ made from the vertices of $T$ except $K$. For any $K \in \mathcal{V}$ we match to it a unique dual sub-domain $A_{K}$ defined by $\overline{A_{K}}=\bigcup_{T \in \mathcal{K}_{\mathcal{T}_{h}}} \overline{A_{K}^{T}}$, where the region $A_{K}^{T}$ is the polygon whose vertices are $x_{T}, K$ 
and the center of edges of $T$ having in common the vertex $K$. Let $\mathcal{E}$ (resp. $\mathcal{E}_{T}$ ) be the set of interfaces (edges) of $\mathcal{A}_{h}$ (resp. of $\mathcal{A}_{h}$ included in $T \in \mathcal{T}_{h}$ ). For a couple of two control volumes $A_{K}$ and $A_{L}$, we define $\sigma_{K L}^{T}:=A_{K} \cap A_{L} \cap T$. The vector $n_{K L}^{T}$ refers to the outward unit normal to the interface $\sigma_{K L}^{T}$ pointed from $A_{K}$ to $A_{L}$. By $\left|A_{K}\right|$ (resp. $\left.\left|\sigma_{K L}^{T}\right|\right)$ we mean the Lebesgue (resp. Lebesgue superficial) measure of the control volume $A_{K}$ (resp. interface $\sigma_{K L}^{T}$ ). One finally refer to Figure 1 for an illustration of the aforementioned geometrical quantities.

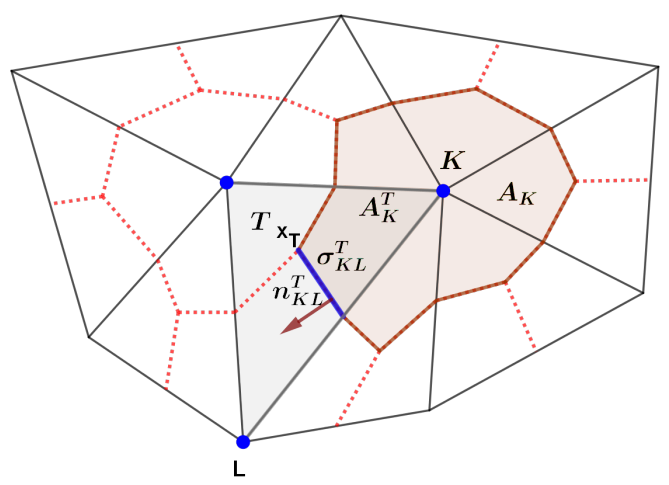

Figure 1: Example of the primal mesh and its corresponding dual mesh.

Remark 2.1. The above definition of the spacial discretizations can be easily adopted to the three-dimensional setting without any constraint. It is sufficient to substitute some terminologies in a proper way. For instance, we would talk about the tetrahedralization instead of triangulation. Then, triangles are replaced by tetrahedral elements. Edges become faces. It is worth mentioning by the way that the mesh-regularity assumption (2.1) still holds regardless the considered dimension.

\subsection{Time discretization}

We will be interested in a one-step discretization of the time derivative of the fluid masses. To this end, we divide the interval $\left(0, t_{f}\right)$ into a uniform subdivision $\left(t^{n}\right)_{n \in \llbracket 0, N \rrbracket}$ whose size is denoted by $\delta t$. Then, $t^{n}=n \delta t$. Without lost of generality, one can take into consideration a non-uniform step $\delta t$.

\subsection{Discrete spaces and unknowns}

The numerical scheme involves two types of meshes. We then need to consider two approximate spaces. The trial space is about the finite volume space denoted by $\widetilde{V}_{h}$ and composed of piecewise constant functions on the control volumes i.e.

$$
\forall \widetilde{u}_{h} \in \widetilde{V}_{h}: \quad \widetilde{u}_{h}=\sum_{K \in \mathcal{V}} u_{K} \mathbf{1}_{\AA_{K}}, \quad\left(u_{K} \in \mathbb{R}, \forall K \in \mathcal{V}\right)
$$

where the $\mathbf{1}_{\AA_{K}}$ is the indicator function of the set $\AA_{K}$ (the topological interior of $A_{K}$ ). Notice that one has $\widetilde{V}_{h} \subset L^{2}(\Omega)$. On the other hand, one defines the $\mathbb{P}_{1}$ finite elements space denoted by $V_{h}$. It incorporates continuous linear piecewise functions per triangles :

$$
V_{h}=\left\{u_{h} \in \mathcal{C}^{0}(\bar{\Omega}), u_{h \mid T} \in \mathbb{P}_{1}, \forall T \in \mathcal{T}_{h}\right\} \subset H^{1}(\Omega)
$$

Then, one writes

$$
\forall u_{h} \in V_{h}: \quad u_{h}=\sum_{K \in \mathcal{V}} u_{K} \varphi_{K}, \quad \nabla u_{h}=\sum_{K \in \mathcal{V}} u_{K} \nabla \varphi_{K}
$$


where $\left(\varphi_{K}\right)_{K \in \mathcal{V}}$ is nothing more than the shape functions basis such that $\varphi_{K}\left(x_{L}\right)=1$ if $K=L$ and $\varphi_{K}\left(x_{L}\right)=0$ otherwise. We recall that $\sum_{K \in \mathcal{V}} \varphi_{K}=1$ which yields $\sum_{K \in \mathcal{V}} \nabla \varphi_{K}=0$. To take into account the boundary conditions prescribed in (1.5), we specify

$$
V_{h}^{0}=\left\{u_{h} \in V_{h}, u_{h}\left(x_{K}\right)=0, \forall K \in \mathcal{V}_{D}\right\} .
$$

In order to have $V_{h}^{0} \subset H_{\Gamma_{D}}^{1}$ we need to assume that $\Gamma_{D} \cap \Gamma_{N}=\{S\} \subset \mathcal{V}_{D}$. In other words, the articulation point of $\Gamma_{D}$ and $\Gamma_{N}$ is a vertex of Dirichlet type. Furthermore, we equip $V_{h}$ by the natural semi-norm :

$$
\left\|u_{h}\right\|_{V_{h}}^{2}=\int_{\Omega}\left|\nabla u_{h}\right|^{2} \mathrm{~d} x, \quad \forall u_{h} \in V_{h} .
$$

We recall that $\|\cdot\|_{V_{h}}$ is indeed a norm $V_{h}^{0}$ by virtue of the discrete Poincaré inequality [16].

As we are dealing with time-dependent functions, we then have to reveal their discrete counterparts. Let $w_{h}^{n+1} \in\left\{u_{h}^{n+1}, \widetilde{u}_{h}^{n+1}\right\}$ for $n \in \llbracket 0, N-1 \rrbracket$, we construct $w_{h, \delta t}$ such that its restriction to the sub-interval $\left(t^{n}, t^{n+1}\right]$ is equal to $w_{h}^{n+1}$. If $F$ is a nonlinear function, its action on $w_{h, \delta t}$ is considered in the interpolation sense. Precisely, the nonlinear finite element interpolation will be denoted by $F_{h}(u) \in V_{h}$ whenever $u_{h}$ belongs to $V_{h}$. Then, one writes

$$
F_{h, \delta t}(u)=\sum_{n=0}^{N-1} \sum_{K \in \mathcal{V}} F\left(u_{K}^{n+1}\right) \varphi_{K} \mathbf{1}_{\left(t^{n}, t^{n+1}\right)} .
$$

Moreover, the finite volume reconstruction for the nonlinear function $F$ is understood in the following sense :

$$
F\left(\widetilde{u}_{h, \delta t}\right)=\sum_{n=0}^{N-1} \sum_{K \in \mathcal{V}} F\left(u_{K}^{n+1}\right) \mathbf{1}_{\AA_{K} \times\left(t^{n}, t^{n+1}\right)} .
$$

For simplicity we employ a permeability tensor which is constant per triangles. Generally, this assumption can be dropped by averaging the matrix $\Lambda^{h} \approx \Lambda$ such that

$$
\left(\Lambda^{h}\right)_{\mid T}=\frac{1}{|T|} \int_{T} \Lambda(x) \mathrm{d} x, \quad \forall T \in \mathcal{T}_{h} .
$$

\section{Finite volume scheme}

Formally, the founding idea of the proposed scheme is to perform a finite volume discretization on the dual mesh while the gradient operator is approximated thanks to a $\mathbb{P}_{1}$ finite element scheme on the primal mesh. In the sequel, we neglect the gravity effects since we are essentially interested in the main terms of model's equations and the contribution corresponding to the gravity terms can be added without technical difficulties. So, to discretize the continuous model (1.1)-(1.5), we integrate its equations on the cell $A_{K} \times\left(t^{n}, t^{n+1}\right]$, we apply the Green formula and we approach the fluxes using adequate approximations of the involved functions. This yields a discrete system whose equations read :

$$
\begin{gathered}
p_{\alpha, K}^{0}=\frac{1}{\left|A_{K}\right|} \int_{A_{K}} p_{\alpha}^{0}(x) \mathrm{d} x, \quad \alpha \in\{g, w\}, \quad \forall K \in \mathcal{V}_{D}^{c} . \\
\phi_{K}\left(\rho_{g}\left(p_{g, K}^{n+1}\right) s_{g, K}^{n+1}-\rho_{g}\left(p_{g, K}^{n}\right) s_{g, K}^{n}\right)-\frac{\delta t}{\left|A_{K}\right|} \sum_{T \in \mathcal{K}_{\mathcal{T}_{h}}} \sum_{L \in \mathcal{V}_{K T}} \rho_{g, K L}^{n+1} M_{g}\left(s_{g, K L}^{n+1}\right) \Lambda_{K L}^{T} \delta_{K L}^{n+1} p_{g} \\
+\delta t \rho_{g}\left(p_{g, K}^{n+1}\right) s_{g, K}^{n+1} f_{P, K}^{n+1}=\delta t \rho_{g}\left(p_{g, K}^{n+1}\right)\left(s_{g, K}^{I}\right)^{n+1} f_{I, K}^{n+1}, \\
\phi_{K}\left(\rho_{w}\left(p_{w, K}^{n+1}\right) s_{w, K}^{n+1}-\rho_{w}\left(p_{w, K}^{n}\right) s_{w, K}^{n}\right)-\frac{\delta t}{\left|A_{K}\right|} \sum_{T \in \mathcal{K}_{\mathcal{T}_{h}}} \sum_{L \in \mathcal{V}_{K T}} \rho_{w, K L}^{n+1} M_{w}\left(s_{w, K L}^{n+1}\right) \Lambda_{K L}^{T} \delta_{K L}^{n+1} p_{w} \\
+\delta t \rho_{w}\left(p_{w, K}^{n+1}\right) s_{w, K}^{n+1} f_{P, K}^{n+1}=\delta t \rho_{w}\left(p_{w, K}^{n+1}\right)\left(s_{w, K}^{I}\right)^{n+1} f_{I, K}^{n+1},
\end{gathered}
$$




$$
\begin{gathered}
s_{g, K}^{n+1}+s_{w, K}^{n+1}=1, \\
p_{c}\left(s_{g, K}^{n+1}\right)=p_{g, K}^{n+1}-p_{w, K}^{n+1}, \quad \forall K \in \mathcal{V}_{D}^{c}, \quad \forall n \in \llbracket 0, N-1 \rrbracket .
\end{gathered}
$$

In the case where $K \in \mathcal{V}_{D}$ we simply sustain the Dirichlet boundary constraint $p_{g, K}^{n}=p_{w, K}^{n}=0$, $\forall n \in \llbracket 0, N \rrbracket$. The notation $\delta_{K L}^{n+1}$ indicates to the differencing operator. For a given function $w$, the latter is defined by

$$
\delta_{K L}^{n+1} w=w_{L}^{n+1}-w_{K}^{n+1} .
$$

Let us now write explicitly the formulas of the coefficients of chief importance appearing in the proposed numerical scheme. Firstly, the density of the phase $\alpha$ is approximated thanks to the following integral mean

$$
\frac{1}{\rho_{\alpha, K L}^{n+1}}:=\left\{\begin{array}{ll}
\frac{1}{p_{\alpha, K}^{n+1}-p_{\alpha, L}^{n+1}} \int_{p_{\alpha, L}^{n+1}}^{p_{\alpha, K}^{n+1}} \frac{1}{\rho_{\alpha}(z)} \mathrm{d} z, & \text { if } p_{\alpha, L}^{n+1} \neq p_{\alpha, K}^{n+1} \\
\frac{1}{\rho_{\alpha}\left(p_{\alpha, K}^{n+1}\right)}, & \text { otherwise }
\end{array} .\right.
$$

Secondly, the coefficient $\Lambda_{K L}^{T}$ stands for the transmissibility or the stiffness coefficient between the control volumes $A_{K}$ and $A_{L}$ in th element $T$

$$
\Lambda_{K L}^{T}=-\int_{T} \Lambda(x) \nabla \varphi_{K} \cdot \nabla \varphi_{L} \mathrm{~d} x=\Lambda_{L K}^{T}
$$

We point out that this number is not necessary nonnegative especially when one considers triangular meshes with some obtuse angles and/or anisotropic permeability tensors. We also recall that one always has

$$
\Lambda_{K K}^{T}=-\sum_{L \in \mathcal{V}_{K T}} \Lambda_{K L}=\int_{T} \Lambda(x) \nabla \varphi_{K} \cdot \nabla \varphi_{K} \mathrm{~d} x>0 .
$$

Contrary to [33], the value of the $\alpha$-phase saturation $s_{\alpha}$ on the interface $\sigma_{K L}^{T}$ is chosen here in a nonstandard way when the transmissibilities are negative

$$
s_{\alpha, K L}^{n+1}:=\left\{\begin{array}{ll} 
\begin{cases}s_{\alpha, L}^{n+1} & \text { if }\left(p_{\alpha, L}^{n+1}-p_{\alpha, K}^{n+1}\right) \geq 0 \\
s_{\alpha, K}^{n+1} & \text { if }\left(p_{\alpha, L}^{n+1}-p_{\alpha, K}^{n+1}\right)<0\end{cases} & \text { if } \Lambda_{K L}^{T} \geq 0 \\
\min _{J \in \mathcal{V}_{T}}\left(s_{\alpha, J}^{n+1}\right) & \text { if } \Lambda_{K L}^{T}<0
\end{array} .\right.
$$

In fact, the above expression of $s_{\alpha, K L}^{n+1}$, especially the last case, allows to dampen the impact of the nonpositive stiffness coefficients on the coercivity of the scheme as we will see later on. It is worth indicating that this choice is symmetric with respect to $K$ and $L$ which means $s_{\alpha, K L}^{n+1}=s_{\alpha, L K}^{n+1}$. This property guarantees the conservativity of the numerical fluxes.

The approximation of the injection and production functions is nothing more than the average of the latter on $A_{K} \times\left(t^{n}, t^{n+1}\right]$. For instance, one sets

$$
f_{q, K}^{n+1}=\frac{1}{\delta t\left|A_{K}\right|} \int_{t^{n}}^{t^{n+1}} \int_{A_{K}} f_{q}(x, t) \mathrm{d} x \mathrm{~d} t, \quad q \in\{I, P\} .
$$

Remark 3.1. The discrete counterpart of (1.7) is defined by

$$
p_{K}^{n+1}=p_{g, K}^{n+1}-\widehat{p}_{g}\left(s_{g, K}^{n+1}\right)=p_{w, K}^{n+1}+\widehat{p}_{w}\left(s_{g, K}^{n+1}\right) \quad \forall K \in \mathcal{V}_{D}^{c}, \quad \forall n \in \llbracket 0, N-1 \rrbracket .
$$

As in Remark 1.1, the compatibility condition $p_{c}(0)=0$ allows

$$
s_{g, K}^{n+1}=0 \text { and } p_{K}^{n+1}=0 \quad \forall K \in \mathcal{V}_{D}, \quad \forall n \in \llbracket 0, N-1 \rrbracket .
$$

Let us thereafter study some remarkable properties of the scheme. 


\section{Fundamental a priori estimates}

In this section we establish that the approximate saturation for each phase fulfills an $L^{\infty}$-bound as well as we prove simultaneous uniform estimates on the gradients of both : the global pressure and the new capillary function. To this purpose, we assume for the moment that the system (3.1)-(3.5) admits a solution denoted $\left(p_{\alpha, h, \delta t}\right)_{\alpha=g, w}$.

Lemma 4.1. (Stability estimate on the saturations) Let $\left(p_{\alpha, h, \delta t}\right)_{\alpha=g, w}$ be a solution to the numerical scheme (3.1)-(3.5). Then, for $\alpha \in\{g, w\}$, the discrete saturation of the $\alpha$-phase obeys its physical bounds i.e.

$$
0 \leq s_{\alpha, K}^{n} \leq 1, \quad \forall K \in \mathcal{V}, \forall n \in \llbracket 0, N \rrbracket
$$

Proof. Let $\alpha=g$. We proceed by induction on the integer $n$ to show that $s_{g, K}^{n} \geq 0$. For $n=0$ this property is an immediate consequence of Assumption $\left(\mathbf{A}_{0}\right)$. Let $n \in \llbracket 1, N-1 \rrbracket$. We now suppose that $s_{g, K}^{l} \geq 0$ for every $l \leq n$ and prove that $s_{g, K}^{n+1} \geq 0$. To this end, we select a vertex $K \in \mathcal{V}_{D}^{c}$ for which $s_{g, K}^{n+1}=\min _{L \in \mathcal{V}_{D}^{c}} s_{g, L}^{n+1}$. Multiplying $(3.2)$ by $\left(s_{g, K}^{n+1}\right)^{-}=-\min \left(s_{g, K}^{n+1}, 0\right)$ implies

$$
\begin{aligned}
\phi_{K}\left(\rho_{g}\left(p_{g, K}^{n+1}\right) s_{g, K}^{n+1}\right. & \left.-\rho_{g}\left(p_{g, K}^{n}\right) s_{g, K}^{n}\right)\left(s_{g, K}^{n+1}\right)^{-} \\
& \overbrace{-\frac{\delta t}{\left|A_{K}\right|} \sum_{T \in \mathcal{K}_{\mathcal{T}_{h}}} \sum_{L \in \mathcal{V}_{K T}} \rho_{g, K L}^{n+1} M_{g}\left(s_{g, K L}^{n+1}\right) \Lambda_{K L}^{T} \delta_{K L}^{n+1} p_{g}\left(s_{g, K}^{n+1}\right)^{-}}^{\operatorname{CONV}_{K}^{n+1}} \\
& +\delta t \rho_{g}\left(p_{g, K}^{n+1}\right) s_{g, K}^{n+1} f_{P, K}^{n+1}\left(s_{g, K}^{n+1}\right)^{-}=\delta t \rho\left(p_{g, K}^{n+1}\right) s_{g, I, K}^{n+1} f_{P, K}^{n+1}\left(s_{g, K}^{n+1}\right)^{-}
\end{aligned}
$$

By the induction assumption on $s_{g, K}^{n}$, the first term of the left hand side of the above equality is nonpositive

$\phi_{K}\left(\rho_{g}\left(p_{g, K}^{n+1}\right) s_{g, K}^{n+1}-\rho_{g}\left(p_{g, K}^{n}\right) s_{g, K}^{n}\right)\left(s_{g, K}^{n+1}\right)^{-}=-\phi_{K}\left(\rho_{g}\left(p_{g, K}^{n+1}\right)\left|\left(s_{g, K}^{n+1}\right)^{-}\right|^{2}+\rho_{g}\left(p_{g, K}^{n}\right) s_{g, K}^{n}\left(s_{g, K}^{n+1}\right)^{-}\right) \leq 0$.

In (4.1), $\mathrm{CONV}_{K}^{n+1}$ denotes the convective contribution multiplied by $\left(s_{g, K}^{n+1}\right)^{-}$. From the definition of $s_{g, K L}^{n+1}$ given in (3.9) one distinguishes two cases.

(i) We first assume that $\Lambda_{K L}^{T} \geq 0$.

- In the case where $p_{g, L}^{n+1}-p_{g, K}^{n+1} \geq 0$ we observe

$$
M_{g}\left(s_{g, K L}^{n+1}\right)=M_{g}\left(s_{g, L}^{n+1}\right) \geq M_{g}\left(s_{g, K}^{n+1}\right),
$$

which implies

$$
\rho_{g, K L}^{n+1}\left(s_{g, K}^{n+1}\right)^{-} M_{g}\left(s_{g, K L}^{n+1}\right) \Lambda_{K L}^{T} \delta_{K L}^{n+1} p_{g} \geq \rho_{g, K L}^{n+1}\left(s_{g, K}^{n+1}\right)^{-} M_{g}\left(s_{g, K}^{n+1}\right) \Lambda_{K L}^{T} \delta_{K L}^{n+1} p_{g}=0,
$$

since $M_{g}$ is nondecreasing and extended by zero on $(-\infty, 0]$.

- Now, if $p_{g, L}^{n+1}-p_{g, K}^{n+1}<0$, one gets

$$
M_{g}\left(s_{g, K L}^{n+1}\right)\left(s_{g, K}^{n+1}\right)^{-}=M_{g}\left(s_{g, K}^{n+1}\right)\left(s_{g, K}^{n+1}\right)^{-}=0 .
$$

(ii) Otherwise, we have $\min _{J \in \mathcal{V}_{T}}\left(s_{g, J}^{n+1}\right)=s_{g, K}^{n+1}$ for every $T \in \mathcal{K}_{\mathcal{T}_{h}}$. Therefore

$$
\rho_{g, K L}^{n+1}\left(s_{g, K}^{n+1}\right)^{-} M_{g}\left(s_{g, K L}^{n+1}\right) \Lambda_{K L}^{T} \delta_{K L}^{n+1} p_{g}=\rho_{g, K L}^{n+1}\left(s_{g, K}^{n+1}\right)^{-} M_{g}\left(s_{g, K}^{n+1}\right) \Lambda_{K L}^{T} \delta_{K L}^{n+1} p_{g}=0,
$$

In both cases, one deduces that $\mathrm{CONV}_{K}^{n+1} \leq 0$. Note that the source production term is also nonpositive, that is

$$
\delta t \rho\left(p_{g, K}^{n+1}\right) s_{g, K}^{n+1} f_{P, K}^{n+1}\left(s_{g, K}^{n+1}\right)^{-}=-\delta t \rho\left(p_{g, K}^{n+1}\right) f_{P, K}^{n+1}\left|\left(s_{g, K}^{n+1}\right)^{-}\right|^{2} \leq 0,
$$


whereas the source injection term is nonnegative. Consequently, one has $\left(s_{g, K}^{n+1}\right)^{-}=0$. Thus, we deduce $s_{g, K}^{n+1} \geq 0$. Similarly, we can check that $s_{w, K}^{n+1} \geq 0$ using this time the liquid equation (3.3). Thanks to the fact that $s_{g, K}^{n+1}+s_{w, K}^{n+1}=1$, we automatically get $s_{g, K}^{n+1}, s_{w, K}^{n+1} \leq 1$. This finishes up the proof.

Subsequently, we dissociate the interfaces of the dual mesh $\mathcal{A}_{h}$ with regards to the sign of the transmissibility coefficients. Let us then write

$$
\mathcal{E}^{+}=\left\{\sigma_{K L}^{T} \in \mathcal{E}: \Lambda_{K L}^{T} \geq 0\right\} .
$$

For each phase we denote $\mathcal{E}_{\alpha}^{+}=\left\{\sigma_{K L}^{T} \in \mathcal{E}^{+}: \Lambda_{K L}^{T} \delta_{K L}^{n+1} p_{\alpha} \leq 0\right\}$.

Lemma 4.2. For every $\sigma_{K L}^{T} \in \mathcal{E}$ and $n \in \llbracket 0, N-1 \rrbracket$, there holds

$$
M_{g_{K L}}^{n+1}+M_{w_{K L}}^{n+1} \geq m_{0} .
$$

If further $\sigma_{K L}^{T} \in \mathcal{E}^{+}$then one has

$$
m_{0}\left(\Lambda_{K L}^{T}\left(\delta_{K L}^{n+1} p\right)^{2}+\Lambda_{K L}^{T}\left(\delta_{K L}^{n+1} \xi\right)^{2}\right) \leq \Lambda_{K L}^{T} M_{g_{K L}}^{n+1}\left(\delta_{K L}^{n+1} p_{g}\right)^{2}+\Lambda_{K L}^{T} M_{w_{K L}}^{n+1}\left(\delta_{K L}^{n+1} p_{w}\right)^{2} .
$$

Proof. The proof of the first inequality is quite straightforward and it can be found, for instance, in [21]. We also extend analogous ideas provided in the same reference so as to establish (4.2), which represents the discrete counterpart of the inequality (1.11). Let us then study the different possible cases as presented in [21].

(i) Let us first look at the case where $\sigma_{K L}^{T} \in \mathcal{E}_{g}^{+}$and $\sigma_{K L}^{T} \notin \mathcal{E}_{w}^{+}$. According to (3.9) one has

$$
s_{g, L}^{n+1} \leq s_{g, K}^{n+1}, \quad \text { and } \quad M_{\alpha, K L}^{n+1}=\max _{\left[s_{g, L}^{n+1}, s_{g, K}^{n+1}\right]} M_{\alpha} .
$$

It follows from the expression of $\widehat{p}_{g}\left(s_{g}\right)$ that there exists some $x_{0} \in\left[s_{g, L}^{n+1}, s_{g, K}^{n+1}\right]$ such that

$$
\delta_{K L}^{n+1} \widehat{p}_{g}=f_{w}\left(x_{0}\right) \delta_{K L}^{n+1} p_{c}, \quad \delta_{K L}^{n+1} \widehat{p}_{w}=f_{g}\left(x_{0}\right) \delta_{K L}^{n+1} p_{c} \quad \text { and } \quad f_{w}\left(x_{0}\right)+f_{g}\left(x_{0}\right)=1,
$$

where $f_{\alpha}$ is the fractional flow function of the $\alpha$-phase defined by

$$
f_{\alpha}\left(s_{\alpha}\right)=\frac{M_{s_{\alpha}}\left(s_{\alpha}\right)}{M\left(s_{g}\right)} .
$$

Based on the definition of the global pressure we find

$$
\begin{aligned}
\left(\delta_{K L}^{n+1} p\right)^{2}= & \left(\delta_{K L}^{n+1} p_{w}+\delta_{K L}^{n+1} \widehat{p}_{w}\right)^{2} \\
= & \left(\delta_{K L}^{n+1} p_{w}\right)^{2}+2 f_{g}\left(x_{0}\right) \delta_{K L}^{n+1} p_{w} \delta_{K L}^{n+1} p_{c}+f_{g}\left(x_{0}\right)^{2}\left(\delta_{K L}^{n+1} p_{c}\right)^{2} \\
= & f_{w}\left(x_{0}\right)\left(\delta_{K L}^{n+1} p_{w}\right)^{2}+f_{g}\left(x_{0}\right)\left(\delta_{K L}^{n+1} p_{w}\right)^{2}+2 f_{g}\left(x_{0}\right) \delta_{K L}^{n+1} p_{w} \delta_{K L}^{n+1} p_{c}+f_{g}\left(x_{0}\right)\left(\delta_{K L}^{n+1} p_{c}\right)^{2} \\
& \quad-f_{g}\left(x_{0}\right) f_{w}\left(x_{0}\right)\left(\delta_{K L}^{n+1} p_{c}\right)^{2} .
\end{aligned}
$$

Thereby

$$
M\left(x_{0}\right)\left(\left(\delta_{K L}^{n+1} p\right)^{2}+\delta_{K L}^{n+1} \widehat{p}_{g} \delta_{K L}^{n+1} \widehat{p}_{w}\right)=M_{w}\left(x_{0}\right)\left(\delta_{K L}^{n+1} p_{w}\right)^{2}+M_{g}\left(x_{0}\right)\left(\delta_{K L}^{n+1} p_{g}\right)^{2} .
$$

Owing to the Cauchy-Schwarz inequality, we finally get

$$
m_{0}\left(\left(\delta_{K L}^{n+1} p\right)^{2}+\left(\delta_{K L}^{n+1} \xi\right)^{2}\right) \leq M_{w_{K L}}^{n+1}\left(\delta_{K L}^{n+1} p_{w}\right)^{2}+M_{g_{K L}}^{n+1}\left(\delta_{K L}^{n+1} p_{g}\right)^{2} .
$$

(ii) The case $\sigma_{K L}^{T} \notin \mathcal{E}_{g}^{+}$and $\sigma_{K L}^{T} \in \mathcal{E}_{w}^{+}$is similar to the point $(i)$. 
(iii) We now consider $\sigma_{K L}^{T} \in \mathcal{E}_{g}^{+} \cap \mathcal{E}_{w}^{+}$. Then, $M_{g_{K L}}^{n+1}=M_{g}\left(s_{g, K}^{n+1}\right)$ and $M_{w_{K L}}^{n+1}=M_{w}\left(s_{w, K}^{n+1}\right)$. It has proven in [21] that

$$
2 f_{g}\left(s_{g, K}^{n+1}\right) \delta_{K L}^{n+1} p_{w} \delta_{K L}^{n+1} p_{c}+f_{g}\left(s_{g, K}^{n+1}\right)\left(\delta_{K L}^{n+1} p_{c}\right)^{2} \geq 2 \delta_{K L}^{n+1} p_{w} \delta_{K L}^{n+1} \widehat{p}_{w}+\delta_{K L}^{n+1} p_{c} \delta_{K L}^{n+1} \widehat{p}_{w} .
$$

As a consequence of $\delta_{K L}^{n+1} p_{c}=\delta_{K L}^{n+1} \widehat{p}_{g}+\delta_{K L}^{n+1} \widehat{p}_{w}$, one writes

$$
\begin{aligned}
f_{w}\left(s_{g, K}^{n+1}\right)\left(\delta_{K L}^{n+1} p_{w}\right)^{2} & +f_{g}\left(s_{g, K}^{n+1}\right)\left(\delta_{K L}^{n+1} p_{w}+\delta_{K L}^{n+1} p_{c}\right)^{2} \\
& \geq\left(\delta_{K L}^{n+1} p_{w}\right)^{2}+2 \delta_{K L}^{n+1} p_{w} \delta_{K L}^{n+1} \widehat{p}_{w}+\left(\delta_{K L}^{n+1} \widehat{p}_{w}\right)^{2}+\delta_{K L}^{n+1} \widehat{p}_{g} \delta_{K L}^{n+1} \widehat{p}_{w} .
\end{aligned}
$$

Multiply each side by $M\left(s_{g, K}^{n+1}\right)$ and use once more the Cauchy-Schwarz inequality to deduce

$$
M\left(s_{g, K}^{n+1}\right)\left(\left(\delta_{K L}^{n+1} p\right)^{2}+\left(\delta_{K L}^{n+1} \xi\right)^{2}\right) \leq M_{g_{K L}}^{n+1}\left(\delta_{K L}^{n+1} p_{g}\right)^{2}+M_{w_{K L}}^{n+1}\left(\delta_{K L}^{n+1} p_{w}\right)^{2} .
$$

Whence, (4.2) is verified.

(iv) The last case $\sigma_{K L}^{T} \notin \mathcal{E}_{g}^{+} \cap \mathcal{E}_{w}^{+}$is similar to the previous one. This completes the proof of Lemma 4.2.

The next lemma shows the equivalence of two norms on the space $V_{h}^{0}$. Its proof is based on elementary calculus of the finite element methodology. We refer to [7, 8] for a detailed demonstration.

Lemma 4.3. There exists a constant $C_{1}>0$ depending only on $\bar{\Lambda}, \underline{\Lambda}$ and $\Theta$ mentioned in (2.1) such that

$$
\sum_{\sigma_{K L}^{T} \in \mathcal{E}_{T}}\left|\Lambda_{K L}^{T}\right|\left(w_{K}-w_{L}\right)^{2} \leq C_{1} \int_{\Omega} \Lambda \nabla w_{T} \cdot \nabla w_{T} \mathrm{~d} x, \quad \forall w_{T}=\sum_{K \in \mathcal{V}_{T}} w_{K} \varphi_{K}, \forall T \in \mathcal{T}_{h} .
$$

In the sequel we set $\mathcal{E}_{T}^{ \pm}=\left\{\sigma_{K L}^{T} \in \mathcal{E}_{T} / \pm \Lambda_{K L}^{T} \geq 0\right\}$. The statement of the preceding result can be reformulated in an equivalent manner as follows.

Lemma 4.4. For every $T \in \mathcal{T}_{h}$, the set $\mathcal{E}_{T}$ contains at most one edge for which the corresponding stiffness coefficient is nonpositive. Moreover, there exists a constant $\eta \in(0,1)$ depending only on $\bar{\Lambda}, \underline{\Lambda}$ and $\Theta$ such that

$0 \leq \sum_{\sigma_{K L}^{T} \in \mathcal{E}_{T}^{-}} \Lambda_{K L}^{T}\left(w_{K}-w_{L}\right)^{2}+(1-\eta) \sum_{\sigma_{K L}^{T} \in \mathcal{E}_{T}^{+}} \Lambda_{K L}^{T}\left(w_{K}-w_{L}\right)^{2}, \quad \forall w_{T}=\sum_{K \in \mathcal{V}_{T}} w_{K} \varphi_{K}, \forall T \in \mathcal{T}_{h}$.

Proof. Assume that there exist two nonpositive stiffness coefficients denoted by $\Lambda_{K L}^{T}$ and $\Lambda_{K O}^{T}$ for example. As a result, one immediately gets $\Lambda_{K L}^{T}+\Lambda_{K O}^{T}=\Lambda_{K K}^{T} \leq 0$. Thus $\Lambda_{K K}^{T}=0$, which contradicts with the original definition of the shape function $\varphi_{K \mid T}$.

Let $T \in \mathcal{T}_{h}$. Then, Lemma 4.3 and the formula

$$
\int_{\Omega} \Lambda \nabla w_{T} \cdot \nabla w_{T} \mathrm{~d} x=\sum_{\sigma_{K L}^{T} \in \mathcal{E}_{T}} \Lambda_{K L}^{T}\left(w_{K}-w_{L}\right)^{2}
$$

leads to

$$
0 \leq\left(1+\frac{1}{C_{1}}\right) \sum_{\sigma_{K L}^{T} \in \mathcal{E}_{T}^{-}} \Lambda_{K L}^{T}\left(w_{K}-w_{L}\right)^{2}+\left(1-\frac{1}{C_{1}}\right) \sum_{\sigma_{K L}^{T} \in \mathcal{E}_{T}^{+}}\left|\Lambda_{K L}^{T}\right|\left(w_{K}-w_{L}\right)^{2} .
$$

Dividing each side by $\left(1+\frac{1}{C_{1}}\right)$ entails

$$
0 \leq \sum_{\sigma_{K L}^{T} \in \mathcal{E}_{T}^{-}} \Lambda_{K L}^{T}\left(w_{K}-w_{L}\right)^{2}+\left(1-\frac{2}{C_{1}+1}\right) \sum_{\sigma_{K L}^{T} \in \mathcal{E}_{T}^{+}}\left|\Lambda_{K L}^{T}\right|\left(w_{K}-w_{L}\right)^{2} .
$$

To conclude, one takes $\eta=\frac{2}{C_{1}+1}<1$. Note this parameter is independent of $T \in \mathcal{T}_{h}$. 
Proposition 4.1. Let $\left(p_{\alpha, h, \delta t}\right)_{\alpha=g, w}$ be a solution to the nonlinear system (3.1)-(3.5). Then, there exists a constant $C_{2}$ independent of the discretization parameters namely $h$ and $\delta t$ such that

$$
\begin{gathered}
\sum_{n=0}^{N-1} \delta t\left(\left\|p_{h}^{n+1}\right\|_{V_{h}}^{2}+\left\|\xi_{h}^{n+1}\right\|_{V_{h}}^{2}\right) \leq C_{2}, \\
\sum_{n=0}^{N-1} \delta t \sum_{T \in \mathcal{T}} \sum_{\sigma_{K L}^{T} \in \mathcal{E}_{T}^{+}} \Lambda_{K L}^{T} M_{g_{K L}}^{n+1}\left(\delta_{K L}^{n+1} p_{g}\right)^{2}+\Lambda_{K L}^{T} M_{w_{K L}}^{n+1}\left(\delta_{K L}^{n+1} p_{w}\right)^{2} \leq C_{2},
\end{gathered}
$$

and

$$
0 \leq \sum_{n=0}^{N-1} \delta t \sum_{T \in \mathcal{T}} \sum_{\sigma_{K L}^{T} \in \mathcal{E}_{T}} \Lambda_{K L}^{T} M_{g_{K L}}^{n+1}\left(\delta_{K L}^{n+1} p_{g}\right)^{2}+\Lambda_{K L}^{T} M_{w_{K L}}^{n+1}\left(\delta_{K L}^{n+1} p_{w}\right)^{2} \leq C_{2}
$$

Proof. Let us first focus on the proof of (4.4). We consider the function $g_{\alpha}\left(p_{\alpha}\right)=\int_{0}^{p_{\alpha}} \frac{1}{\rho_{\alpha}(b)} \mathrm{d} b$. We multiply the discrete mass conservation equation for the $\alpha$-phase by $\left|A_{K}\right| g_{\alpha}\left(p_{\alpha, K}^{n+1}\right)$, which is a concave function, and we add them together. We then sum up the result on $K \in \mathcal{V}$ and $n \in \llbracket 0, N-1 \rrbracket$ to obtain

where we write

$$
\Upsilon_{1}+\Upsilon_{2}+\Upsilon_{3}=0
$$

$$
\begin{aligned}
& \Upsilon_{1}=\sum_{\alpha \in\{g, w\}} \sum_{n=0}^{N-1} \sum_{K \in \mathcal{V}}\left|A_{K}\right| \phi_{K}\left(\rho_{\alpha}\left(p_{\alpha, K}^{n+1}\right) s_{\alpha, K}^{n+1}-\rho_{\alpha}\left(p_{\alpha, K}^{n}\right) s_{\alpha, K}^{n}\right) g_{\alpha}\left(p_{\alpha, K}^{n+1}\right), \\
& \Upsilon_{2}=-\sum_{\alpha \in\{g, w\}} \sum_{n=0}^{N-1} \delta t \sum_{K \in \mathcal{V}} \sum_{T \in \mathcal{K}_{\mathcal{T}_{h}}} \sum_{L \in \mathcal{V}_{K T}} \rho_{\alpha, K L}^{n+1} \Lambda_{K L}^{T} M_{\alpha K L}^{n+1}\left(\delta_{K L}^{n+1} p_{\alpha}\right) g_{\alpha}\left(p_{\alpha, K}^{n+1}\right), \\
& \Upsilon_{3}=\sum_{\alpha \in\{g, w\}} \sum_{n=0}^{N-1} \delta t \sum_{K \in \mathcal{V}}\left|A_{K}\right| \rho_{\alpha}\left(p_{\alpha, K}^{n+1}\right)\left(s_{\alpha, K}^{n+1} f_{P, K}^{n+1}-\left(s_{\alpha, K}^{I}\right)^{n+1} f_{I, K}^{n+1}\right) g_{\alpha}\left(p_{\alpha, K}^{n+1}\right) .
\end{aligned}
$$

To begin with, we want to determine a lower bound on $\Upsilon_{1}$. To do that, we introduce the feature the following functions $\mathcal{H}_{\alpha}\left(p_{\alpha}\right)=\rho_{\alpha}\left(p_{\alpha}\right) g_{\alpha}\left(p_{\alpha}\right)-p_{\alpha}, \mathcal{P}_{c}\left(s_{w}\right)=\int_{0}^{s_{w}} p_{c}(1-u) \mathrm{d} u$. It can be easily seen that the first function is nonnegative and vanishes at zero while the second one is concave. Thanks to this information, it has shown in [27] that : for all $s_{\alpha}, s_{\alpha}^{*} \geq 0$ with $s_{g}+s_{w}=s_{g}^{*}+s_{w}^{*}=1$ and $p_{\alpha}, p_{\alpha}^{*} \in \mathbb{R}$ one has

$$
\sum_{\alpha \in\{g, w\}}\left(\rho_{\alpha}\left(p_{\alpha}\right) s_{\alpha}-\rho_{\alpha}\left(p_{\alpha}^{*}\right) s_{\alpha}^{*}\right) g_{\alpha}\left(p_{\alpha}\right) \geq\left(\sum_{\alpha \in\{g, w\}} \mathcal{H}_{\alpha}\left(p_{\alpha}\right) s_{\alpha}-\mathcal{H}_{\alpha}\left(p_{\alpha}^{*}\right) s_{\alpha}^{*}\right)-\mathcal{P}_{c}\left(s_{w}\right)+\mathcal{P}_{c}\left(s_{w}^{*}\right)
$$

As a result of this inequality and Lemma 4.1 one estimates $\Upsilon_{1}$ as follows

$$
-\Upsilon_{1} \leq \underbrace{\phi_{1}\left(\left\|\mathcal{P}_{c}\left(\widetilde{s_{w, h}^{0}}\right)+\sum_{\alpha \in\{g, w\}} \mathcal{H}_{\alpha}\left(\widetilde{p_{\alpha, h}^{0}}\right) \widetilde{s_{\alpha, h}^{0}}\right\|_{L^{1}(\Omega)}\right)}_{C_{0}}<+\infty .
$$

We next treat the hyperbolic term. So, rearranging and gathering the expression of $\Upsilon_{2}$ by edges gives

$$
\Upsilon_{2}=\sum_{\alpha \in\{g, w\}} \sum_{n=0}^{N-1} \delta t \sum_{T \in \mathcal{T}} \sum_{\sigma_{K L}^{T} \in \mathcal{E}_{T}} \rho_{\alpha, K L}^{n+1} \Lambda_{K L}^{T} M_{\alpha K L}^{n+1} \delta_{K L}^{n+1} p_{\alpha} \delta_{K L}^{n+1} g_{\alpha}\left(p_{\alpha}\right)
$$


In light of (3.6) there holds

$$
\Upsilon_{2}=\sum_{\alpha \in\{g, w\}} \sum_{n=0}^{N-1} \delta t \sum_{T \in \mathcal{T}} \sum_{\sigma_{K L}^{T} \in \mathcal{E}_{T}} \Lambda_{K L}^{T} M_{\alpha_{K L}}^{n+1}\left(\delta_{K L}^{n+1} p_{\alpha}\right)^{2} .
$$

We denote

$$
\Upsilon_{2}^{T, n+1}=\sum_{\alpha \in\{g, w\}} \sum_{\sigma_{K L}^{T} \in \mathcal{E}_{T}} \Lambda_{K L}^{T} M_{\alpha_{K L}}^{n+1}\left(\delta_{K L}^{n+1} p_{\alpha}\right)^{2}
$$

which we decompose as follows

$$
\Upsilon_{2}^{T, n+1}=\eta\left(\sum_{\alpha \in\{g, w\}} \sum_{\sigma_{K L}^{T} \in \mathcal{E}_{T}^{+}}\left|\Lambda_{K L}^{T}\right| M_{\alpha_{K L}}^{n+1}\left(\delta_{K L}^{n+1} p_{\alpha}\right)^{2}\right)+\Upsilon_{2, *}^{T, n+1}
$$

where we set

$$
\Upsilon_{2, *}^{T, n+1}=\sum_{\alpha \in\{g, w\}}\left((1-\eta) \sum_{\sigma_{K L}^{T} \in \mathcal{E}_{T}^{+}}\left|\Lambda_{K L}^{T}\right| M_{\alpha_{K L}}^{n+1}\left(\delta_{K L}^{n+1} p_{\alpha}\right)^{2}+\sum_{\sigma_{K L}^{T} \in \mathcal{E}_{T}^{-}} \Lambda_{K L}^{T} M_{\alpha_{K L}}^{n+1}\left(\delta_{K L}^{n+1} p_{\alpha}\right)^{2}\right) .
$$

Thanks to the crucial choice of (3.9) we obtain

$$
\Upsilon_{2, *}^{T, n+1} \geq \sum_{\alpha \in\{g, w\}} \min _{J \in \mathcal{V}_{T}}\left(M_{\alpha}\left(s_{\alpha, J}^{n+1}\right)\right)\left((1-\eta) \sum_{\sigma_{K L} \in \mathcal{E}^{+}}\left|\Lambda_{K L}^{T}\right|\left(\delta_{K L}^{n+1} p_{\alpha}\right)^{2}+\sum_{\sigma_{K L} \in \mathcal{E}^{-}} \Lambda_{K L}^{T}\left(\delta_{K L}^{n+1} p_{\alpha}\right)^{2}\right) .
$$

Now Lemma 4.4 ensures that $\Upsilon_{2, *}^{T, n+1} \geq 0$. At this stage we can observe that $\Upsilon_{2} \geq 0$. This estimate is enhanced thanks to Lemma 4.2. Indeed, combining this latter, the integration by parts formula, and the coercivity of the tensor $\Lambda$ we find

$$
\begin{aligned}
\Upsilon_{2} & \geq \eta \sum_{\alpha \in\{g, w\}} \sum_{n=0}^{N-1} \delta t \sum_{T \in \mathcal{T}} \sum_{\sigma_{K L}^{T} \in \mathcal{E}_{T}^{+}} \Lambda_{K L}^{T} M_{\alpha_{K L}}^{n+1}\left(\delta_{K L}^{n+1} p_{\alpha}\right)^{2} \\
& \geq \eta m_{0} \sum_{n=0}^{N-1} \delta t \sum_{T \in \mathcal{T}} \sum_{\sigma_{K L}^{T} \in \mathcal{E}_{T}} \Lambda_{K L}^{T}\left(\left(\delta_{K L}^{n+1} p\right)^{2}+\left(\delta_{K L}^{n+1} \xi\right)^{2}\right) \\
& \geq \eta m_{0} \underline{\Lambda} \sum_{n=0}^{N-1} \delta t\left(\left\|p_{h}^{n+1}\right\|_{V_{h}}^{2}+\left\|\xi_{h}^{n+1}\right\|_{V_{h}}^{2}\right) .
\end{aligned}
$$

Following [33] there exists $C_{1}^{\prime}$ and $C_{2}^{\prime}$ depending only $\Omega, t_{f}, f_{P}, f_{I}$ such that

$$
\left|\Upsilon_{3}\right| \leq C_{1}^{\prime}\left(\sum_{n=0}^{N-1} \delta t\left\|p_{h}^{n+1}\right\|_{V_{h}}^{2}\right)^{1 / 2}+C_{2}^{\prime}
$$

Using Young's inequality, $a b \leq \varepsilon a^{2}+\frac{1}{4 \varepsilon} b^{2}$, with $\varepsilon=\frac{\eta m_{0} \underline{\Lambda}}{2}$ implies

$$
\left|\Upsilon_{3}\right| \leq \frac{\eta m_{0} \underline{\Lambda}}{2}\left(\sum_{n=0}^{N-1} \delta t\left\|p_{h}^{n+1}\right\|_{V_{h}}^{2}\right)+\frac{2 C_{1}^{\prime 2}}{\eta m_{0} \underline{\Lambda}}+C_{2}^{\prime} .
$$

Let us set $C_{2}=\frac{2}{\eta m_{0} \underline{\Lambda}}\left(\frac{2 C^{\prime 2}}{\eta m_{0} \underline{\Lambda}}+C_{2}^{\prime}+C_{0}\right)$. As a consequence of (4.7), (4.8), (4.10) and (4.11) we ultimately obtain

$$
\sum_{n=0}^{N-1} \delta t\left(\left\|p_{h}^{n+1}\right\|_{V_{h}}^{2}+\left\|\xi_{h}^{n+1}\right\|_{V_{h}}^{2}\right) \leq C_{2} .
$$


Finally, assertions (4.5)-(4.6) result from the previous inequality together with (4.7) and (4.11). Therefore, the proof is concluded.

Corollary 4.1. Keeping the statement of Proposition 4.1, there holds :

$$
\sum_{n=0}^{N-1} \delta t\left\|\mathcal{B}_{h}^{n+1}\right\|_{V_{h}}^{2} \leq C_{3}
$$

and

$$
\begin{aligned}
& \sum_{n=0}^{N-1} \delta t \sum_{T \in \mathcal{T}} \sum_{\sigma_{K L}^{T} \in \mathcal{E}_{T}}\left|\Lambda_{K L}^{T}\right| M_{g_{K L}}^{n+1}\left(\delta_{K L}^{n+1} \widehat{p}_{g}\right)^{2} \leq C_{4}, \\
& \sum_{n=0}^{N-1} \delta t \sum_{T \in \mathcal{T}} \sum_{\sigma_{K L}^{T} \in \mathcal{E}_{T}}\left|\Lambda_{K L}^{T}\right| M_{w_{K L}}^{n+1}\left(\delta_{K L}^{n+1} \widehat{p}_{w}\right)^{2} \leq C_{5} .
\end{aligned}
$$

Proof. It can be easily seen from Lemma 4.3 that

$$
\sum_{n=0}^{N-1} \delta t\left\|\mathcal{B}_{h}^{n+1}\right\|_{V_{h}}^{2} \leq C_{1}\left\|M_{g} M_{w}\right\|_{\infty} \sum_{n=0}^{N-1} \delta t\left\|\xi_{h}^{n+1}\right\|_{V_{h}}^{2} \leq C_{3}=C_{1} C_{2}\left\|M_{g} M_{w}\right\|_{\infty}
$$

Now, the left hand side of (4.13) is dived into terms :

$$
\begin{aligned}
\sum_{n=0}^{N-1} \delta t \sum_{T \in \mathcal{T}} \sum_{\sigma_{K L}^{T} \in \mathcal{E}_{T}}\left|\Lambda_{K L}^{T}\right| M_{g_{K L}}^{n+1}\left(\delta_{K L}^{n+1} \widehat{p}_{g}\right)^{2} & =\overbrace{\sum_{n=0}^{N-1} \delta t \sum_{T \in \mathcal{T}} \sum_{\sigma_{K L}^{T} \in \mathcal{E}_{T}^{-}}\left|\Lambda_{K L}^{T}\right| M_{g_{K L}}^{n+1}\left(\delta_{K L}^{n+1} \widehat{p}_{g}\right)^{2}}^{\mathcal{S}^{1}} \\
& +\underbrace{\sum_{n=0}^{N-1} \delta t \sum_{T \in \mathcal{T}} \sum_{\sigma_{K L}^{T} \in \mathcal{E}_{T}^{+}}\left|\Lambda_{K L}^{T}\right| M_{g_{K L}}^{n+1}\left(\delta_{K L}^{n+1} \widehat{p}_{g}\right)^{2}}_{\mathcal{S}^{2}} .
\end{aligned}
$$

By virtue of (3.9), we get

$$
\mathcal{S}^{1} \leq C_{1}\left\|M_{w}\right\|_{\infty} \sum_{n=0}^{N-1} \delta t\left\|\xi_{h}^{n+1}\right\|_{V_{h}}^{2} \leq C_{3}^{\prime}
$$

Following [32] we estimate $\mathcal{S}^{2}$ with

$$
\mathcal{S}^{2} \leq \sum_{n=0}^{N-1} \delta t \sum_{T \in \mathcal{T}} \sum_{\sigma_{K L}^{T} \in \mathcal{E}_{T}^{+}} \Lambda_{K L}^{T}\left(M_{g_{K L}}^{n+1}\left(\delta_{K L}^{n+1} p_{g}\right)^{2}+M_{w_{K L}}^{n+1}\left(\delta_{K L}^{n+1} p_{w}\right)^{2}\right) \leq C_{4}^{\prime}
$$

Then $C_{4}=C_{3}^{\prime}+C_{4}^{\prime}$. To prove the inequality (4.14) we mimic the same steps.

We mention without proof the existence property of discrete solutions to the numerical scheme (3.1)-(3.5). Certainly, this statement can be shown thanks to a fixed point result, see for instance [17], based on the above energy estimates and the discrete maximum principle. We refer to [23] for more information on the demonstration.

Proposition 4.2. There exists at least one solution to the nonlinear system (3.1)-(3.5). 


\section{Convergence analysis}

In this section we first present some relevant properties which are somehow mandatory for the convergence of the proposed numerical scheme. To this purpose, we set $\widetilde{\mathcal{U}}_{\alpha, h, \delta t}=\rho_{\alpha}\left(\widetilde{p}_{\alpha, h, \delta t}\right) \tilde{s}_{\alpha, h, \delta t}$ and $\mathcal{U}_{\alpha, h, \delta t}=\rho_{\alpha, h, \delta t}\left(p_{\alpha}\right) s_{\alpha, h, \delta t}$. Using the same arguments exhibited in [23] we can establish straightforwardly that the underlined quantities enjoy similar patterns when the discretization steps tend to zero.

Lemma 5.1. For $\alpha \in\{g, w\}$ there holds

$$
\lim _{h, \delta t \rightarrow 0}\left\|\tilde{\mathcal{U}}_{\alpha, h, \delta t}-\mathcal{U}_{\alpha, h, \delta t}\right\|_{L^{1}\left(Q_{t_{f}}\right)}=0 .
$$

The following lemma states classical compactness results namely the space and time translates on the sequence $\left(\widetilde{\mathcal{U}}_{\alpha, h, \delta t}\right)$.

Lemma 5.2. (Compactness properties) Let $\left(p_{\alpha, h, \delta t}\right)_{\alpha=g, w}$ be a solution to the nonlinear numerical scheme (3.1)-(3.5). Then, the following estimates hold

$$
\int_{\Omega^{\prime} \times\left(0, t_{f}\right)}\left|\tilde{\mathcal{U}}_{\alpha, h, \delta t}(x+y, t)-\tilde{\mathcal{U}}_{\alpha, h, \delta t}(x, t)\right| \mathrm{d} x \mathrm{~d} t \leq \omega_{\alpha}(|y|),
$$

where for every $y \in \mathbb{R}^{d}$ we set $\Omega^{\prime}=\{x \in \Omega,[x, x+y] \subset \Omega\}$ with $\omega_{\alpha}$ is a modulus of continuity such that $\omega_{\alpha}(|y|) \rightarrow 0$ as $|y| \rightarrow 0$, and

$$
\int_{\Omega \times\left(0, t_{f}-\tau\right)}\left|\widetilde{\mathcal{U}}_{\alpha, h, \delta t}(x, t+\tau)-\widetilde{\mathcal{U}}_{\alpha, h, \delta t}(x, t)\right|^{2} \mathrm{~d} x \mathrm{~d} t \leq \omega_{\alpha}^{*}(\tau),
$$

such that the modulus of continuity $\omega_{\alpha}^{*}(\tau)$ tends to 0 as $\tau \rightarrow 0$.

The demonstration of this lemma is omitted because it mimics standard ideas as stressed in $[3,6,21]$. In fact, the proof of inequality (5.2) leans essentially on the uniform estimate (4.2) as conducted for instance in [23], whereas that of (5.3) is more intricate and it involves classical arguments as addressed in [21] and later on in $[3,6]$.

Theorem 5.1. Under assumptions $\left(\boldsymbol{A}_{0}\right)-\left(\boldsymbol{A}_{7}\right)$, let $\left(\mathcal{T}_{h}\right)$ be a sequence of meshes to $\Omega$ such that the shape regularity condition (2.1) holds. Let $\left(p_{\alpha, h, \delta t}\right)_{\alpha=g, w}$ be the corresponding family of discrete solutions to the numerical scheme (3.1)-(3.5). Then, as $(h, \delta t)$ goes to $(0,0)$, the following convergences hold up to a subsequence

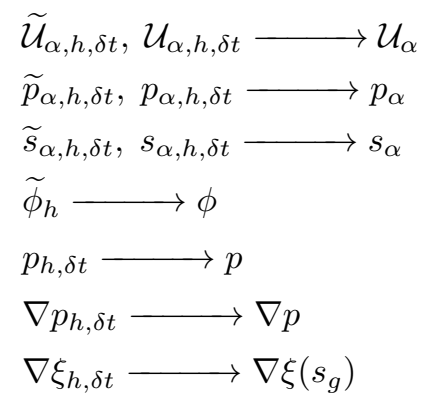

$$
\begin{aligned}
& \text { strongly in } L^{1}\left(Q_{t_{f}}\right) \text { and a.e in } Q_{t_{f}} \text {, } \\
& \text { a.e. in } Q_{t_{f}} \text {, } \\
& \text { a.e. in } Q_{t_{f}} \text {, } \\
& \text { a.e. in } \Omega \text {, } \\
& \text { weakly in } L^{2}\left(Q_{t_{f}}\right) \text {, } \\
& \text { weakly in } L^{2}\left(0, t_{f} ; L^{2}(\Omega)^{d}\right) \text {, } \\
& \text { weakly in } L^{2}\left(0, t_{f} ; L^{2}(\Omega)^{d}\right) \text {. }
\end{aligned}
$$

Furthermore, one has

$$
0 \leq s_{\alpha} \leq 1 \quad \text { and } \quad \mathcal{U}_{\alpha}=\rho_{\alpha}\left(p_{\alpha}\right) s_{\alpha} \quad \text { a.e in } Q_{t_{f}}
$$

Finally, the pair of pressures $\left(p_{g}\right)_{\alpha=g, w}$ is a weak solution to the mathematical model (1.1)-(1.5) in the sense of Definition 1.1. 
Proof. The strong convergence of $\widetilde{\mathcal{U}}_{\alpha, h, \delta t}$ towards $\mathcal{U}_{\alpha}$ (up to a subsequence) is ensured by the space and the time translation estimates on the underlined sequence together with the Fréchet-Kolmogorov compactness theorem [20]. The finite element reconstruction tends to the same limit by virtue of Lemma 5.1. Let us next show the assertions (5.5) and (5.6) simultaneously.

Following [27], we define the map $\mathfrak{F}:\left(p_{g}, s_{g}\right) \in \mathbb{R} \times[0,1] \longrightarrow\left(\mathcal{R}_{g}, \mathcal{R}_{w}\right) \in \mathfrak{F}(\mathbb{R} \times[0,1]) \subset$ $\left[0, \phi_{1} \rho_{1}\right]^{2}$ where

$$
\mathcal{R}_{g}=\rho_{g}\left(p_{g}\right) s_{g}, \quad \mathcal{R}_{w}=\rho_{w}\left(p_{g}-p_{c}\left(s_{g}\right)\right)\left(1-s_{g}\right) .
$$

Computing the determinant of the Jacobian matrix of $\mathfrak{F}$ yields

$$
\begin{aligned}
d \mathfrak{F}\left(p_{g}, s_{g}\right)=\operatorname{det}\left[\begin{array}{cc}
\frac{\partial}{\partial p_{g}} \rho_{g}\left(p_{g}\right) s_{g} & \frac{\partial}{\partial s_{g}} \rho_{g}\left(p_{g}\right) s_{g} \\
\frac{\partial}{\partial p_{g}} \rho_{w}\left(p_{g}-p_{c}\left(s_{g}\right)\right)\left(1-s_{g}\right) & \frac{\partial}{\partial s_{g}} \rho_{w}\left(p_{g}-p_{c}\left(s_{g}\right)\right)\left(1-s_{g}\right)
\end{array}\right] \\
=-\left(\rho_{g}^{\prime}\left(p_{g}\right) s_{g}\left(p_{c}^{\prime}\left(s_{g}\right) \rho_{w}^{\prime}\left(p_{g}-p_{c}\left(s_{g}\right)\right)\left(1-s_{g}\right)+\rho_{w}\left(p_{g}-p_{c}\left(s_{g}\right)\right)\right)\right. \\
\left.+\rho_{w}^{\prime}\left(p_{g}-p_{c}\left(s_{g}\right)\right)\left(1-s_{g}\right) \rho_{g}\left(p_{g}\right)\right)<0, \quad \forall\left(p_{g}, s_{g}\right) \in \mathbb{R} \times[0,1] .
\end{aligned}
$$

Additionally, $\mathfrak{F}$ is one-to-one. To see this, we should study the different possible cases as follows. Let us take $s_{g}^{1}, s_{g}^{2} \in[0,1]$ and $p_{g}^{1}, p_{g}^{2} \in \mathbb{R}$.

(i) First, we assume that $s_{g}^{1}=s_{g}^{2} \neq 0$ and $p_{g}^{1} \neq p_{g}^{2}$. Then, one has $\rho_{g}\left(p_{g}^{1}\right) \neq \rho_{g}\left(p_{g}^{2}\right)$ since $\rho_{g}$ is increasing. Consequently, we get $\mathcal{R}_{g}^{1} \neq \mathcal{R}_{g}^{2}$. Otherwise, if $s_{g}^{1}=s_{g}^{2}=0$, we obtain $\mathcal{R}_{w}^{1}=\rho_{w}\left(p_{g}^{1}\right) \neq \mathcal{R}_{w}^{2}=\rho_{w}\left(p_{g}^{2}\right)$ by the monotony of $\rho_{w}$.

(ii) Now, we suppose that $s_{g}^{1} \neq s_{g}^{2}$ and $p_{g}^{1}=p_{g}^{2}$. This gives $\rho_{g}\left(p_{g}^{1}\right)=\rho_{g}\left(p_{g}^{2}\right)$. Then, $\mathcal{R}_{g}^{1} \neq \mathcal{R}_{g}^{2}$.

(iii) Finally, we look at the last case $s_{g}^{1} \neq s_{g}^{2}$ and $p_{g}^{1} \neq p_{g}^{2}$. The treatment of the situations $\left(s_{g}^{1}=0\right.$ and $\left.s_{g}^{2}=1\right)$ or $\left(s_{g}^{1}=1\right.$ and $\left.s_{g}^{2}=0\right)$ is analogous to the point (ii). Hence, we take $s_{g}^{1}, s_{g}^{2}$ belonging to the open segment $(0,1)$. Suppose by absurd that $\mathcal{R}_{g}^{1}=\mathcal{R}_{g}^{2}$ and $\mathcal{R}_{w}^{1}=\mathcal{R}_{w}^{2}$. Considering that $s_{g}^{1}<s_{g}^{2}$ yields

$$
\frac{1-s_{g}^{2}}{1-s_{g}^{1}}<1, \quad \text { and } \quad \frac{s_{g}^{1}}{s_{g}^{2}}<1
$$

Therefore, the relationships

$$
\rho_{g}\left(p_{g}^{1}\right) \frac{s_{g}^{1}}{s_{g}^{2}}=\rho_{g}\left(p_{g}^{2}\right), \quad \rho_{w}\left(p_{g}^{1}-p_{c}\left(s_{g}^{1}\right)\right)=\frac{1-s_{g}^{2}}{1-s_{g}^{1}} \rho_{w}\left(p_{g}^{2}-p_{c}\left(s_{g}^{2}\right)\right),
$$

imply

$$
\rho_{g}\left(p_{g}^{1}\right)>\rho_{g}\left(p_{g}^{2}\right), \quad \rho_{w}\left(p_{g}^{1}-p_{c}\left(s_{g}^{1}\right)\right)<\rho_{w}\left(p_{g}^{2}-p_{c}\left(s_{g}^{2}\right)\right) .
$$

Since $\rho_{g}$ and $\rho_{w}$ are increasing functions we deduce

$$
p_{g}^{1}>p_{g}^{2}, \quad p_{g}^{1}-p_{c}\left(s_{g}^{1}\right)<p_{g}^{2}-p_{c}\left(s_{g}^{2}\right) .
$$

Subtracting these inequalities leads to

$$
p_{c}\left(s_{g}^{1}\right)>p_{c}\left(s_{g}^{2}\right)
$$

The fact that the capillary function $p_{c}$ is also increasing gives a contradiction. Ultimately, the case $s_{g}^{1}<s_{g}^{2}$ is similar. 
Consequently, the map $\mathfrak{F}$ is indeed an injection. On the other hand, the function $\mathfrak{F}$ is onto by construction. Hence, the global inversion theorem ensures the fact that $\mathfrak{F}$ is a diffeomorphism. Now, in view of (5.4) and the continuity of $\mathfrak{F}^{-1}$ we infer

$$
\begin{aligned}
& \widetilde{p}_{\alpha, h, \delta t}, p_{\alpha, h, \delta t} \longrightarrow p_{\alpha} \quad \text { a.e. in } Q_{t_{f}} \text {, } \\
& \widetilde{s}_{\alpha, h, \delta t}, s_{\alpha, h, \delta t} \longrightarrow s_{\alpha} \quad \text { a.e. in } Q_{t_{f}} \text {. }
\end{aligned}
$$

Accordingly, one has $0 \leq s_{\alpha} \leq 1, \mathcal{U}_{\alpha}=\rho_{\alpha}\left(p_{\alpha}\right) s_{\alpha}$ a.e. in $Q_{t_{f}}$. We recall that $\widetilde{\phi}_{h}$ is defined as the average value of the porosity within each control volume $A_{K}$ of $\mathcal{A}_{h}$. Thanks to Assumption $\left(\mathbf{A}_{1}\right)$ there holds

$$
\widetilde{\phi}_{h} \longrightarrow \phi \quad \text { a.e. in } \Omega,
$$

which establishes (5.7). Owing to (4.4), the sequence $\left\{\nabla p_{h, \delta t}\right\}$ remains bounded in $L^{2}\left(0, t_{f} ; L^{2}(\Omega)^{d}\right)$. Then there exists $G \in L^{2}\left(0, t_{f} ; L^{2}(\Omega)^{d}\right)$ such that

$$
\nabla p_{h, \delta t} \longrightarrow G \text { weakly in } L^{2}\left(0, t_{f} ; L^{2}(\Omega)^{d}\right),
$$

up to another subsequence if needed. Moreover, the Poincaré inequality implies that $\left\{p_{h, \delta t}\right\}$ is uniformly bounded in $L^{2}\left(Q_{t_{f}}\right)$. Whence, one can find a $p$ in the underlined space such that

$$
p_{h, \delta t} \longrightarrow p \text { weakly in } L^{2}\left(Q_{t_{f}}\right) .
$$

So, the identification of the limits is a direct consequence of the conforming nature of the $\mathbb{P}_{1}$ finite elements. Thus, $\nabla p=G$ in the sense of distributions and $p \in L^{2}\left(0, t_{f} ; H_{\Gamma_{D}}^{1} \Omega\right)$. Thereby, the proof of (5.8)-(5.9) is concluded. One mimics a similar fashion to draw the weak convergence (5.10) for the capillary term. In light of (1.8), the strong convergence (5.6) on the saturations, Assumptions $\left(\mathbf{A}_{2}\right)$ and $\left(\mathbf{A}_{6}\right)$ we entail that the discrete correction terms given in the relationships (1.7) converge to $\widehat{p}_{g}\left(s_{g}\right), \widehat{p}_{w}\left(s_{g}\right)$ almost everywhere in $Q_{t_{f}}$ and strongly in $L^{2}\left(Q_{t_{f}}\right)$. Then, it follows that $p_{\alpha} \in L^{2}\left(Q_{t_{f}}\right)$.

Let us now prove that $\left(p_{g}, p_{w}\right)$ is a weak solution to the problem (1.1)-(1.5) in the sense of Definition 1.1. Let us then provide the proof in the case where $\alpha=g$. The proof of the case $\alpha=w$ follows in the same fashion. To this purpose, let $\psi \in \mathcal{C}_{c}^{\infty}\left(\bar{\Omega} \times\left[0, t_{f}\right)\right)$, with $\psi=0$ on $\Gamma_{D} \times\left[0, t_{f}\right)$. We multiply the $g$-phase discrete equation (3.2) by $\left|A_{K}\right| \psi_{K}^{n+1}:=\left|A_{K}\right| \psi\left(x_{K}, t^{n+1}\right)$, for all $K \in \mathcal{V}$ and $n \in \llbracket 0, N-1 \rrbracket$. We sum up all the equations of the resulting system to get

$$
\mathcal{D}_{1}^{h, \delta t}+\mathcal{D}_{2}^{h, \delta t}+\mathcal{D}_{3}^{h, \delta t}=0
$$

where we write

$$
\begin{aligned}
\mathcal{D}_{1}^{h, \delta t} & =\sum_{n=0}^{N-1} \sum_{K \in \mathcal{V}}\left|A_{K}\right| \phi_{K}\left(\rho_{g}\left(p_{g, K}^{n+1}\right) s_{g, K}^{n+1}-\rho_{g}\left(p_{g, K}^{n}\right) s_{g, K}^{n}\right) \psi_{K}^{n+1}, \\
\mathcal{D}_{2}^{h, \delta t} & =-\sum_{n=0}^{N-1} \delta t \sum_{K \in \mathcal{V}} \sum_{T \in \mathcal{K}_{\mathcal{T}_{h}}} \sum_{L \in \mathcal{V}_{K T}} \rho_{g, K L}^{n+1} \Lambda_{K L}^{T} M_{g_{K L}}^{n+1}\left(\delta_{K L}^{n+1} p_{g}\right) \psi_{K}^{n+1}, \\
\mathcal{D}_{3}^{h, \delta t} & =\sum_{n=0}^{N-1} \delta t \sum_{K \in \mathcal{V}}\left|A_{K}\right| \rho_{g}\left(p_{g, K}^{n+1}\right)\left(s_{g, K}^{n+1} f_{P, K}^{n+1}-\left(s_{g, K}^{I}\right)^{n+1} f_{I, K}^{n+1}\right) \psi_{K}^{n+1} .
\end{aligned}
$$

We perform a discrete integration by parts in time to find that

$$
\begin{aligned}
\mathcal{D}_{1}^{h, \delta t} & =-\sum_{n=0}^{N-1} \sum_{K \in \mathcal{V}} \int_{A_{K}} \phi_{K} \rho_{g}\left(p_{g, K}^{n}\right) s_{g, K}^{n} \int_{t^{n}}^{t^{n+1}} \partial_{t} \psi\left(x_{K}, t\right) \mathrm{d} x \mathrm{~d} t \\
& -\sum_{K \in \mathcal{V}} \int_{A_{K}} \phi_{K} \rho_{g}\left(p_{g, K}^{0}\right) s_{g, K}^{0} \psi\left(x_{K}, 0\right) \mathrm{d} x .
\end{aligned}
$$


By virtue of (5.4), (5.7), (5.11) and the smoothness of the test function $\psi$ we infer

$$
\lim _{h, \delta t \rightarrow 0} \mathcal{D}_{1}^{h, \delta t}=-\int_{Q_{t_{f}}} \phi \rho_{g}\left(p_{g}\right) s_{g} \partial_{t} \psi \mathrm{d} x \mathrm{~d} t-\int_{\Omega} \rho_{g}\left(p_{g}^{0}\right) s_{g}^{0} \psi(\cdot, 0) \mathrm{d} x .
$$

We now treat the convergence of the convective contribution. Since we have no estimate on the gradient of the gas pressure, we are led to pass to the limit through the artificial decomposition of $p_{g}$. This splits $\mathcal{D}_{2}^{h, \delta t}$ into two terms

$$
\mathcal{D}_{2}^{h, \delta t}=\mathcal{D}_{2,1}^{h, \delta t}+\mathcal{D}_{2,2}^{h, \delta t},
$$

where we consider

$$
\begin{aligned}
\mathcal{D}_{2,1}^{h, \delta t} & =-\sum_{n=0}^{N-1} \delta t \sum_{K \in \mathcal{V}} \sum_{T \in \mathcal{K}_{\mathcal{T}_{h}}} \sum_{L \in \mathcal{V}_{K T}} \rho_{g, K L}^{n+1} \Lambda_{K L}^{T} M_{g_{K L}}^{n+1}\left(\delta_{K L}^{n+1} p\right) \psi_{K}^{n+1} \\
& =\sum_{n=0}^{N-1} \delta t \sum_{K \in \mathcal{V}} \sum_{T \in \mathcal{K}_{\mathcal{T}_{h}}} \sum_{\sigma_{K L}^{T} \in \mathcal{E}_{T}} \rho_{g, K L}^{n+1} \Lambda_{K L}^{T} M_{g_{K L}}^{n+1} \delta_{K L}^{n+1} p \delta_{K L}^{n+1} \psi, \\
\mathcal{D}_{2,2}^{h, \delta t} & =-\sum_{n=0}^{N-1} \delta t \sum_{K \in \mathcal{V}} \sum_{T \in \mathcal{K}_{\mathcal{T}_{h}}} \sum_{L \in \mathcal{V}_{K T}} \rho_{g, K L}^{n+1} \Lambda_{K L}^{T} M_{g_{K L}}^{n+1}\left(\delta_{K L}^{n+1} \widehat{p}_{g}\right) \psi_{K}^{n+1} \\
& =\sum_{n=0}^{N-1} \delta t \sum_{K \in \mathcal{V}} \sum_{T \in \mathcal{K}_{\mathcal{T}_{h}}} \sum_{\sigma_{K L}^{T} \in \mathcal{E}_{T}} \rho_{g, K L}^{n+1} \Lambda_{K L}^{T} M_{g_{K L}}^{n+1} \delta_{K L}^{n+1} \widehat{p}_{g} \delta_{K L}^{n+1} \psi
\end{aligned}
$$

In oder to study the convergence of the above expressions, we need to introduce the piecewise functions (keeping in mind that $w \in\left\{p_{g}, s_{g}\right\}$ )

$$
\begin{aligned}
& \bar{w}_{T}^{n+1}:=\max _{x \in T} w_{h}^{n+1}, \quad \underline{w}_{T}^{n+1}:=\min _{x \in T} w_{h}^{n+1} . \\
& \bar{w}_{h, \delta t_{\mid T \times\left(t^{n}, t^{n+1}\right]}}:=\underline{w}_{T}^{n+1}, \quad \underline{w}_{h, \delta t_{\mid T \times\left(t^{n}, t^{n+1}\right]}}:=\bar{w}_{T}^{n+1} .
\end{aligned}
$$

Let us begin with the investigation of the limit of $\mathcal{D}_{2,1}^{h, \delta t}$. To this end, we further define

$$
\mathcal{C}_{2,1}^{h, \delta t}=-\sum_{n=0}^{N-1} \delta t \sum_{K \in \mathcal{V}} \sum_{T \in \mathcal{K}_{\mathcal{T}_{h}}} \sum_{L \in \mathcal{V}_{K T}} \rho_{g}\left(\underline{p}_{g, T}^{n+1}\right) \Lambda_{K L}^{T} M_{g}\left(\underline{s}_{g, T}^{n+1}\right) \delta_{K L}^{n+1} p \psi_{K}^{n+1}
$$

The integration by parts formula allows to rewrite $\mathcal{C}_{2,1}^{h, \delta t}$ in the integral form

$$
\mathcal{C}_{2,1}^{h, \delta t}=\int_{Q_{t_{f}}} \rho_{g}\left(\underline{p}_{g, h, \delta t}\right) M_{g}\left(\underline{s}_{g, h, \delta t}\right) \nabla p_{h, \delta t} \cdot \nabla \psi_{h, \delta t} \mathrm{~d} x \mathrm{~d} t .
$$

Our goal hereafter is to make sure that

$$
\underline{s}_{g, h, \delta t} \longrightarrow s_{g}, \quad \underline{p}_{g, h, \delta t} \longrightarrow p_{g} \text { a.e. in } Q_{t_{f}} .
$$

Following $[8,33]$ one gets

$$
\int_{Q_{t_{f}}}\left|\xi\left(\bar{s}_{g, h, \delta t}\right)-\xi\left(\underline{s}_{g, h, \delta t}\right)\right|^{2} \mathrm{~d} x \mathrm{~d} t \leq C_{5}^{\prime} h \sum_{n=0}^{N-1} \delta t\left\|\xi_{h}^{n+1}\right\|_{V_{h}}^{2} \leq C_{6}^{\prime} h \longrightarrow 0, \text { as } h, \delta t \longrightarrow 0 .
$$

Up to the extraction of a new subsequence, one deduces

$$
\left|\xi\left(\bar{s}_{g, h, \delta t}\right)-\xi\left(\underline{s}_{g, h, \delta t}\right)\right| \longrightarrow 0 \quad \text { a.e. in } Q_{t_{f}} .
$$


It follows from the inequality $\xi\left(\underline{s}_{g, h, \delta t}\right) \leq \xi\left(s_{g, h, \delta t}\right) \leq \xi\left(\bar{s}_{g, h, \delta t}\right)$, and (5.6) that

$$
\bar{s}_{g, h, \delta t}, \underline{s}_{g, h, \delta t} \longrightarrow s_{g} \quad \text { a.e. in } Q_{t_{f}} .
$$

On the other hand

$$
\frac{1}{2} \int_{Q_{t_{f}}}\left|\bar{p}_{g, h, \delta t}-\underline{p}_{g, h, \delta t}\right|^{2} \mathrm{~d} x \mathrm{~d} t \leq \int_{Q_{t_{f}}}\left|\bar{p}_{h, \delta t}-\underline{p}_{h, \delta t}\right|^{2} \mathrm{~d} x \mathrm{~d} t+\int_{Q_{t_{f}}}\left|\widehat{\widehat{p}}_{g, h, \delta t}-\underline{\underline{p}}_{g, h, \delta t}\right|^{2} \mathrm{~d} x \mathrm{~d} t .
$$

The first integral of the right hand side of (5.14) can be estimated by

$$
\int_{Q_{t_{f}}}\left|\bar{p}_{h, \delta t}-\underline{p}_{h, \delta t}\right|^{2} \mathrm{~d} x \mathrm{~d} t \leq C_{7}^{\prime} h \sum_{n=0}^{N-1} \delta t\left\|p_{h}^{n+1}\right\|_{V_{h}}^{2} \leq C_{8}^{\prime} h \longrightarrow 0, \text { as } h, \delta t \longrightarrow 0 .
$$

In addition, one has

$$
\int_{Q_{t_{f}}}\left|\overline{\widehat{p}}_{g, h, \delta t}-\underline{\widehat{p}}_{g, h, \delta t}\right|^{2} \mathrm{~d} x \mathrm{~d} t \leq C_{9}^{\prime} \int_{Q_{t_{f}}}\left|\bar{s}_{g, h, \delta t}-\underline{s}_{g, h, \delta t}\right|^{2} \mathrm{~d} x \mathrm{~d} t \longrightarrow 0, \text { as } h, \delta t \longrightarrow 0 .
$$

Accordingly, one extracts another unlabeled subsequence if needed to obtain

$$
\bar{p}_{g, h, \delta t}, \underline{p}_{g, h, \delta t} \longrightarrow p_{g} \quad \text { a.e. in } Q_{t_{f}} .
$$

Now, as a consequence of the Lebesgue dominated convergence theorem (LDCT) we claim

$$
\lim _{h, \delta t \rightarrow 0} \mathcal{C}_{2,1}^{h, \delta t}=\int_{Q_{t_{f}}} \rho_{g}\left(p_{g}\right) M_{g}\left(s_{g}\right) \nabla p \cdot \nabla \psi \mathrm{d} x \mathrm{~d} t
$$

We next check that

$$
\lim _{h, \delta t \rightarrow 0}\left|\mathcal{C}_{2,1}^{h, \delta t}-\mathcal{D}_{2,1}^{h, \delta t}\right|=0
$$

We compute the difference

$$
\left|\mathcal{C}_{2,1}^{h, \delta t}-\mathcal{D}_{2,1}^{h, \delta t}\right| \leq\left\|M_{g}\right\|_{\infty} \mathcal{R}_{2,1}^{h, \delta t}+\left\|\rho_{g}\right\|_{\infty} \mathcal{X}_{2,1}^{h, \delta t}
$$

where

$$
\begin{gathered}
\mathcal{R}_{2,1}^{h, \delta t}=\sum_{n=0}^{N-1} \delta t \sum_{K \in \mathcal{V}} \sum_{T \in \mathcal{K}_{\mathcal{T}_{h}}} \sum_{\sigma_{K L}^{T} \in \mathcal{E}_{T}}\left|\rho_{g}\left(\bar{p}_{g, h, \delta t}\right)-\rho_{g}\left(\underline{p}_{g, h, \delta t}\right)\right|\left|\Lambda_{K L}^{T}\right|\left|\delta_{K L}^{n+1} p\right|\left|\delta_{K L}^{n+1} \psi\right|, \\
\mathcal{X}_{2,1}^{h, \delta t}=\sum_{n=0}^{N-1} \delta t \sum_{K \in \mathcal{V}} \sum_{T \in \mathcal{K}_{\mathcal{T}_{h}}} \sum_{\sigma_{K L}^{T} \in \mathcal{E}_{T}}\left|M_{g}\left(\bar{s}_{g, h, \delta t}\right)-M_{g}\left(\underline{s}_{g, h, \delta t}\right)\right|\left|\Lambda_{K L}^{T}\right|\left|\delta_{K L}^{n+1} p\right|\left|\delta_{K L}^{n+1} \psi\right| .
\end{gathered}
$$

In light of the smoothness of the function $\psi$, the regularity of the mesh and the Cauchy-Schwarz inequality we discover

$$
\begin{aligned}
\mathcal{R}_{2,1}^{h, \delta t} \leq C_{10}^{\prime} & \left(\sum_{n=0}^{N-1} \delta t \sum_{K \in \mathcal{V}} \sum_{T \in \mathcal{K}_{\mathcal{T}_{h}}} \sum_{\sigma_{K L}^{T} \in \mathcal{E}_{T}}|T|\left|\rho_{g}\left(\bar{p}_{g, T}^{n+1}\right)-\rho_{g}\left(\underline{p}_{g, T}^{n+1}\right)\right|^{2}\right)^{\frac{1}{2}} \\
& \times\left(\sum_{n=0}^{N-1} \delta t \sum_{K \in \mathcal{V}} \sum_{T \in \mathcal{K}_{\mathcal{T}_{h}}} \sum_{\sigma_{K L}^{T} \in \mathcal{E}_{T}}\left|\Lambda_{K L}^{T}\right|\left|\delta_{K L}^{n+1} p\right|^{2}\right)^{\frac{1}{2}}
\end{aligned}
$$


Using Lemma 4.3, the a priori estimate (4.4), the almost everywhere convergence

$$
\rho_{g}\left(\bar{p}_{g, h, \delta t}\right), \rho_{g}\left(\underline{p}_{g, h, \delta t}\right) \longrightarrow \rho_{g}\left(p_{g}\right) \quad \text { a.e. in } Q_{t_{f}},
$$

and the LDCT, we ensure that

$$
\begin{aligned}
\mathcal{R}_{2,1}^{h, \delta t} & \leq C_{11}^{\prime}\left(\int_{Q_{t_{f}}}\left|\rho_{g}\left(\bar{p}_{g, h, \delta t}\right)-\rho_{g}\left(\underline{p}_{g, h, \delta t}\right)\right|^{2} \mathrm{~d} x \mathrm{~d} t\right)^{\frac{1}{2}}\left(\sum_{n=0}^{N-1} \delta t\left\|p_{h}^{n+1}\right\|_{V_{h}}^{2}\right)^{\frac{1}{2}} \\
& \leq C_{12}^{\prime} h \longrightarrow 0, \text { as } h, \delta t \longrightarrow 0 .
\end{aligned}
$$

The same conclusion can be drawn for $\mathcal{X}_{2,1}^{h, \delta t}$ i.e.

$$
\mathcal{X}_{2,1}^{h, \delta t} \leq C_{13}^{\prime} h \longrightarrow 0, \text { as } h, \delta t \longrightarrow 0
$$

In conclusion, one has $\lim _{h, \delta t \rightarrow 0}\left|\mathcal{C}_{2,1}^{h, \delta t}-\mathcal{D}_{2,1}^{h, \delta t}\right|=0$. Now, to conclude the convergence of $\mathcal{D}_{2}^{h, \delta t}$ it remains to establish that

$$
\lim _{h, \delta t \rightarrow 0} \mathcal{D}_{2,2}^{h, \delta t}=\int_{Q_{t_{f}}} \rho_{g}\left(p_{g}\right) \nabla \mathcal{B}\left(s_{g}\right) \cdot \nabla \psi \mathrm{d} x \mathrm{~d} t .
$$

To this purpose we set

$$
\mathcal{C}_{2,2}^{h, \delta t}=\sum_{n=0}^{N-1} \delta t \sum_{K \in \mathcal{V}} \sum_{T \in \mathcal{K}_{\mathcal{T}_{h}}} \sum_{\sigma_{K L} \in \mathcal{E}_{\mathcal{T}}} \rho_{g, K L}^{n+1} \Lambda_{K L}^{T} \delta_{K L}^{n+1} \mathcal{B}\left(s_{g}\right) \delta_{K L}^{n+1} \psi_{K}^{n+1}
$$

Using similar arguments as conducted for $\mathcal{C}_{2,1}^{h, \delta t}$ together with the weak convergence (5.10), we can easily pass to the limit in the above expression and claim

$$
\lim _{h, \delta t \rightarrow 0} \mathcal{C}_{2,2}^{h, \delta t}=\int_{Q_{t_{f}}} \rho_{g}\left(p_{g}\right) \nabla \mathcal{B}\left(s_{g}\right) \cdot \nabla \psi \mathrm{d} x \mathrm{~d} t
$$

Let us prove that

$$
\lim _{h, \delta t \rightarrow 0}\left|\mathcal{C}_{2,2}^{h, \delta t}-\mathcal{D}_{2,2}^{h, \delta t}\right|=0
$$

For this, we consider $z_{K L}^{n+1} \in\left[\min \left(s_{g, K}^{n+1}, s_{g, L}^{n+1}\right), \max \left(s_{g, K}^{n+1}, s_{g, L}^{n+1}\right)\right]$ such that

$$
\begin{aligned}
\sqrt{M_{g}\left(z_{K L}^{n+1}\right)} \int_{s_{g, K}^{n+1}}^{s_{g, L}^{n+1}} \frac{\sqrt{M_{w}(1-u)}}{M(u)} p_{c}^{\prime}(u) \mathrm{d} u & =\int_{s_{g, K}^{n+1}}^{s_{g, L}^{n+1}} \frac{\sqrt{M_{g}(u) M_{w}(1-u)}}{M(u)} p_{c}^{\prime}(u) \mathrm{d} u \\
& :=\delta_{K L}^{n+1} \xi .
\end{aligned}
$$

Similarly, let $w_{K L}^{n+1} \in\left[\min \left(s_{w, K}^{n+1}, s_{w, L}^{n+1}\right), \max \left(s_{w, K}^{n+1}, s_{w, L}^{n+1}\right)\right]$ verifying

$$
\sqrt{M_{w}\left(w_{K L}^{n+1}\right)} \int_{s_{g, K}^{n+1}}^{s_{g, L}^{n+1}} \frac{\sqrt{M_{w}(1-u)}}{M(u)} p_{c}^{\prime}(u) \mathrm{d} u=\int_{s_{g, K}^{n+1}}^{s_{g, L}^{n+1}} \frac{M_{w}(1-u)}{M(u)} p_{c}^{\prime}(u) \mathrm{d} u:=\delta_{K L}^{n+1} \widehat{p}_{g} .
$$

According to (5.15)-(5.16) we observe that

$$
\sqrt{M_{g}\left(z_{K L}^{n+1}\right)} \delta_{K L}^{n+1} \widehat{p}_{g}=\sqrt{M_{w}\left(w_{K L}^{n+1}\right)} \delta_{K L}^{n+1} \xi .
$$


Owing to this crucial relationship and the Cauchy-Schwarz inequality, we deduce

$$
\begin{aligned}
& \left|\mathcal{C}_{2,2}^{h, \delta t}-\mathcal{D}_{2,2}^{h, \delta t}\right| \leq \underbrace{\sum_{n=0}^{N-1} \delta t \sum_{K \in \mathcal{V}} \sum_{T \in \mathcal{K}_{\mathcal{T}_{h}}} \sum_{\sigma_{K L}^{T} \in \mathcal{E}_{T}}\left|\Lambda_{K L}^{T}\right|\left|\sqrt{M_{g}\left(z_{K L}^{n+1}\right) M_{w}\left(w_{K L}^{n+1}\right)} \delta_{K L}^{n+1} \xi-\delta_{K L}^{n+1} \mathcal{B}\right|\left|\delta_{K L}^{n+1} \psi\right|}_{\mathcal{Y}^{h, \delta t}} \\
& +\sum_{n=0}^{N-1} \delta t \sum_{K \in \mathcal{V}} \sum_{T \in \mathcal{K}_{\mathcal{T}_{h}}} \sum_{\sigma_{K L}^{T} \in \mathcal{E}_{T}}\left|M_{g}\left(z_{K L}^{n+1}\right)-M_{g_{K L}}^{n+1}\right|\left|\Lambda_{K L}^{T}\right|\left|\delta_{K L}^{n+1} \widehat{p}_{g}\right|\left|\delta_{K L}^{n+1} \psi\right| \\
& \leq \mathcal{Y}^{h, \delta t}+\left(\sum_{n=0}^{N-1} \delta t \sum_{K \in \mathcal{V}} \sum_{T \in \mathcal{K}_{\mathcal{T}_{h}}} \sum_{\sigma_{K L}^{T} \in \mathcal{E}_{T}}\left|\Lambda_{K L}^{T}\right|\left|M_{g}\left(z_{K L}^{n+1}\right)+M_{g_{K L}}^{n+1}\right|\left|\delta_{K L}^{n+1} \widehat{p}_{g}\right|^{2}\right)^{\frac{1}{2}} \\
& \times(\underbrace{\sum_{n=0}^{N-1} \delta t \sum_{K \in \mathcal{V}} \sum_{T \in \mathcal{K}_{\mathcal{T}_{h}}} \sum_{\sigma_{K L}^{T} \in \mathcal{E}_{T}}\left|M_{g}\left(z_{K L}^{n+1}\right)-M_{g_{K L}}^{n+1}\right|\left|\Lambda_{K L}^{T}\right|\left|\delta_{K L}^{n+1} \psi\right|^{2}}_{\mathcal{Z}^{h, \delta t}})^{\frac{1}{2}} \\
& \leq \mathcal{Y}^{h, \delta t}+C_{13}^{\prime} \mathcal{Z}^{h, \delta t} \text {. }
\end{aligned}
$$

Let us now estimate $\mathcal{Y}^{h, \delta t}$ and $\mathcal{Z}^{h, \delta t}$. On the one hand, the function $\sqrt{\left(M_{g} M_{w}\right) \circ \xi^{-1}}$ is uniformly continuous on the compact $[\xi(0), \xi(1)]$. As a consequence, there exists a bounded modulus of continuity $\omega$ of the same function such that (see e.g. [8])

$$
\left|\sqrt{M_{g}\left(z_{K L}^{n+1}\right) M_{w}\left(w_{K L}^{n+1}\right)} \delta_{K L}^{n+1} \xi-\delta_{K L}^{n+1} \mathcal{B}\right| \leq \omega\left(\bar{\xi}_{T}^{n+1}-\underline{\xi}_{T}^{n+1}\right)\left|\delta_{K L}^{n+1} \xi\right| .
$$

Apply once again the Cauchy-Schwarz inequality to infer

$$
\begin{aligned}
\mathcal{Y}^{h, \delta t} & \leq\left(\sum_{n=0}^{N-1} \delta t \sum_{K \in \mathcal{V}} \sum_{T \in \mathcal{K}_{\mathcal{T}_{h}}} \sum_{\sigma_{K L}^{T} \in \mathcal{E}_{T}}\left|\Lambda_{K L}^{T}\right|\left|\delta_{K L}^{n+1} \xi\right|^{2}\right)^{\frac{1}{2}} \\
& \times\left(\sum_{n=0}^{N-1} \delta t \sum_{K \in \mathcal{V}} \sum_{T \in \mathcal{K}_{\mathcal{T}_{h}}} \omega\left(\bar{\xi}_{T}^{n+1}-\underline{\xi}_{T}^{n+1}\right)^{2} \sum_{\sigma_{K L}^{T} \in \mathcal{E}_{T}}\left|\Lambda_{K L}^{T}\right|\left|\delta_{K L}^{n+1} \psi\right|^{2}\right)^{\frac{1}{2}} .
\end{aligned}
$$

On the other hand, Lemma 4.3 entails the existence of a constant $C_{14}^{\prime}$ such that

$$
\sum_{\sigma_{K L}^{T} \in \mathcal{E}_{T}}\left|\Lambda_{K L}^{T}\right|\left|\delta_{K L}^{n+1} \psi\right|^{2} \leq C_{14}^{\prime}|T|
$$

Therefore, one finds

$$
\mathcal{Y}^{h, \delta t} \leq C_{15}^{\prime}\left(\int_{Q_{t_{f}}}\left|\omega\left(\xi\left(\bar{s}_{g, h, \delta t}\right)-\xi\left(\underline{s}_{g, h, \delta t}\right)\right)\right|^{2} \mathrm{~d} x \mathrm{~d} t\right)^{\frac{1}{2}} .
$$

The function $\omega$ is continuous and bounded such that $\omega(0)=0$. In addition, the sequence $\left(\xi\left(\bar{s}_{g, h, \delta t}\right)-\xi\left(\underline{s}_{g, h, \delta t}\right)\right)$ is bounded and converges almost everywhere towards 0 . As a result

$$
\lim _{h, \delta t \rightarrow 0} \mathcal{Y}^{h, \delta t}=0
$$

Taking into account Corollary 4.1, we follow an analogous reasoning to check that

$$
\lim _{h, \delta t \rightarrow 0} \mathcal{Z}^{h, \delta t}=0 .
$$


Hence, the convergence proof of the convective term is finished. Finally, bearing in mind the strong convergence of the gas saturation and and the a.e convergence of the gas pressure, we apply the LDCT to demonstrate that

$$
\lim _{h, \delta t \rightarrow 0} \mathcal{D}_{3}^{h, \delta t}=\int_{Q_{t_{f}}} \rho_{g}\left(p_{g}\right)\left(s_{g} f^{P}-s_{g}^{I} f^{I}\right) \psi \mathrm{d} x \mathrm{~d} t .
$$

This concludes the proof of Theorem 5.1.

\section{Numerical validation}

We now show the effectiveness and the robustness of the proposed method by numerical experiments. In the all tests below, we indicate that the nonlinear system coming from the numerical discretization is solved by the Newton-Raphson procedure. The stopping criterion is fixed to $10^{-10}$. The porous medium fills the quadrangle $\Omega=(0, L x) \times(0, L y)$. The open $\Omega$ is discretized with a refined sequence of triangular meshes taken from the benchmark [25]. The angles of the triangulation are acute which permits to include the case of nonnegative transmissibilities whenever the tensor $\Lambda$ is the identity matrix. The latter property is evidently lost in anisotropic occurrences.

\subsection{Error assessment of the method}

We first begin by illustrating the efficiency of the suggested approach for solving a nonlinear degenerate parabolic equation through several selected exact solutions taken from $[8,9]$. To this end, let us then consider the academic problem

$$
\left\{\begin{array}{ll}
\partial_{t} s-\operatorname{div}(a(s) \Lambda \nabla f(s))=0 & \text { in } \Omega \times\left(0, t_{f}\right) \\
s(\cdot, 0)=s^{0} & \text { in } \Omega
\end{array},\right.
$$

subject to a Dirichlet's boundary condition corresponding to the trace of the considered analytical solution. The nonlinear diffusion function $a$ is continuous, nonincreasing and degenerate at $s=0$. We assume that $a$ is extended by zero for all $s<0$. Here, the permeability tensor $\Lambda$ is chosen as a homogeneous diagonal matrix

$$
\Lambda=\left[\begin{array}{cc}
\lambda_{x} & 0 \\
0 & \lambda_{y}
\end{array}\right] .
$$

The proposed finite volume scheme for the discretization of the system (6.1) reads

$$
s_{K}^{0}=\frac{1}{\left|A_{K}\right|} \int_{A_{K}} s^{0}(x) \mathrm{d} x, \quad \forall K \in \mathcal{V},
$$

and $\forall K \in \mathcal{V}_{D}^{c}, \forall n \in \llbracket 0, N-1 \rrbracket$

$$
s_{K}^{n+1}-s_{K}^{n}-\frac{\delta t}{\left|A_{K}\right|} \sum_{T \in \mathcal{K}_{\mathcal{T}_{h}}} \sum_{L \in \mathcal{V}_{K T}} \Lambda_{K L}^{T} a_{K L}^{T}\left(f\left(s_{L}^{n+1}\right)-f\left(s_{K}^{n+1}\right)\right)=0 .
$$

Contrary to the upwind scheme (3.9), at the interface $\sigma_{K L}^{T}$, the nonlinear diffusion coefficient $a_{K L}^{T}$ is here centered expect for the negative case of the transmissibility

$$
a_{K L}^{T}:=\left\{\begin{array}{ll}
\frac{a\left(s_{K}^{n+1}\right)+a\left(s_{L}^{n+1}\right)}{2} & \text { if } \Lambda_{K L}^{T} \geq 0 \\
a\left(\min _{J \in \mathcal{V}_{T}}\left(s_{J}^{n+1}\right)\right) & \text { if } \Lambda_{K L}^{T}<0
\end{array} .\right.
$$

This idea has been inspired from the work [8]. Notice that $a_{K L}^{T}=a_{L K}^{T}$ regardless the sign of $\Lambda_{K L}^{T}$. Since the angles of the simplices are acute we automatically have $\Lambda_{K L}^{T} \geq 0$ for constant 
eigenvalues $\lambda_{x}=\lambda_{y}$. We indicate that in [8] the authors proposed an upwind based scheme for $a_{K L}^{T}$. The reason behind the crucial choice (6.5) is twofolds : it first consists in reducing the impact of the numerical diffusion and therefore getting a better convergence rate when the discretization parameters tend to zero. This expectation is confirmed numerically as the tables below exhibit. On a second place the expression of (6.5), especially the last case, guarantees the coercivity-like property as highlighted in the proof of Proposition 4.1.

Theoretically, the scheme is positivity-preserving and convergent. A proof on the first property is detailed in Lemma A.1. For the convergence results, it is sufficient to proceed as in [8, 10]. Now, to evaluate the error between the analytical solution and the discrete one we compute the norms :

$$
\mathcal{N}_{h, \delta t}^{r}=\left\|s-\widetilde{s}_{h, \delta t}\right\|_{L^{r}\left(Q_{t_{f}}\right)}, \quad \text { for } r=1,2, \infty .
$$

In the sequel of this subsection, the final time is set to $t_{f}=0.2$. The time step is proportional to the square of the mesh size $h$. We also set $L x=L y=1$, then $\Omega=(0,1)^{2}$.

\subsubsection{Example 1: Heat equation in a nonlinear form}

In this first example we compare the approximate solution provided by the numerical scheme (6.3)-(6.4) with the exact solution :

$$
s_{e}(x, y, t)=\frac{\cos (\pi x) e^{-\pi^{2} \lambda_{x} t}+1}{2}, \quad \forall(x, y, t) \in \Omega \times\left(0, t_{f}\right),
$$

in the sense of the norm $\mathcal{N}_{h, \delta t}^{r}$. The nonlinearities used in the test are :

$$
a(s)=s \text { and } f(s)=\log (s) .
$$

By substituting (6.6) into (6.1) we find the classical heat equation. In the next two tables we list the obtained results for two different situations of the tensor $\Lambda$ given in (6.2). In Table 1 we have considered the isotropic permeability matrix leading to $\lambda_{x}=\lambda_{y}=1$ while in Table 2 we have taken $\lambda_{x}=1$ and $\lambda_{y}=10^{3}$. It can be seen that the method is accurate of second order in the isotropic case whereas its convergence rate falls down to 1 in the anisotropic case. Indeed, this is caused by the semi-upwind scheme (6.5) together with the influence of medium's anisotropy. On the other hand, the saturation $\widetilde{s}_{h, \delta t}$ remains positive which a crucial gain. This fact also sustains the robustness of the proposed discretization for the preservation of such a bound.

\begin{tabular}{|c||c|c|c|c|c|c||c|}
\hline $\mathrm{h}$ & $\mathcal{N}_{h, \delta t}^{2}$ & Rate & $\mathcal{N}_{h, \delta t}^{1}$ & Rate & $\mathcal{N}_{h, \delta t}^{\infty}$ & Rate & $\min \widetilde{s}_{h, \delta t}$ \\
\hline 0.250 & 0.547 E-03 & - & 0.144 E-03 & - & 0.495 E-02 & - & 0.299 E-01 \\
0.125 & 0.213 E-03 & 1.364 & 0.615 E-04 & 1.232 & 0.195 E-02 & 1.344 & 0.765 E-02 \\
0.063 & 0.620 E-04 & 1.802 & 0.189 E-04 & 1.725 & 0.604 E-03 & 1.709 & 0.195 E-02 \\
0.031 & 0.163 E-04 & 1.881 & 0.508 E-05 & 1.850 & 0.191 E-03 & 1.622 & 0.474 E-03 \\
0.016 & 0.420 E-05 & 2.052 & 0.132 E-05 & 2.037 & 0.511 E-04 & 1.995 & 0.126 E-03 \\
\hline
\end{tabular}

Table 1: Numerical errors for the first example with $\lambda_{x}=\lambda_{y}=1$.

\subsubsection{Example 2: Porous medium equation with a smooth solution}

We now test our scheme on the well-known porous medium equation corresponding to

$$
a(s)=2 s \text { and } f(s)=s .
$$

In this second case, we consider its classical solution in two dimensions given by

$$
s_{e}(x, y, t)=\frac{\left(1 / 16 \lambda_{x}\right)(x-0.5)^{2}+\left(1 / 16 \lambda_{y}\right)(y-0.5)^{2}}{1-t}, \quad \forall(x, y, t) \in \Omega \times\left(0, t_{f}\right) .
$$




\begin{tabular}{|c||c|c|c|c|c|c||c|}
\hline $\mathrm{h}$ & $\mathcal{N}_{h, \delta t}^{2}$ & Rate & $\mathcal{N}_{h, \delta t}^{1}$ & Rate & $\mathcal{N}_{h, \delta t}^{\infty}$ & Rate & $\min \widetilde{s}_{h, \delta t}$ \\
\hline 0.250 & 0.468 E-02 & - & 0.124 E-02 & - & 0.418 E-01 & - & 0.299 E-01 \\
0.125 & 0.242 E-02 & 0.950 & 0.686 E-03 & 0.858 & 0.232 E-01 & 0.851 & 0.765 E-02 \\
0.063 & 0.126 E-02 & 0.955 & 0.341 E-03 & 1.020 & 0.149 E-01 & 0.646 & 0.195 E-02 \\
0.031 & 0.659 E-03 & 0.914 & 0.167 E-03 & 1.005 & 0.103 E-01 & 0.515 & 0.474 E-03 \\
0.016 & 0.349 E-03 & 0.961 & 0.831 E-04 & 1.058 & 0.681 E-02 & 0.627 & 0.126 E-03 \\
\hline
\end{tabular}

Table 2: Numerical errors for the first example with $\lambda_{x}=1$ and $\lambda_{y}=1000$.

Tables 3-4 show the errors following the norms $\mathcal{N}_{h, \delta t}^{r}$ and their corresponding convergence rates respectively. In Table 3 we implemented an identical permeability with $\lambda_{x}=\lambda_{y}=1$. As predicted, a super-convergence is achieved as well as the discrete solution respects its physical range. The situation is slightly different for the anisotropic problem where $\lambda_{x}=1$ and $\lambda_{y}=10^{-5}$ as displayed in Table 4. The method based on the choice (6.5) loses its second order accuracy with respect to the anisotropy of the permeability. Nonetheless, it is still of order "one" even if the ratio $\lambda_{x} / \lambda_{y}$ is important. Otherwise, this latter has no effect on the positiveness of $\widetilde{s}_{h, \delta t}$, which is a fundamental point as it is needed below in case of the compressible diphasic flow to select at least one admissible solution.

\begin{tabular}{|c||c|c|c|c|c|c||c|}
\hline $\mathrm{h}$ & $\mathcal{N}_{h, \delta t}^{2}$ & Rate & $\mathcal{N}_{h, \delta t}^{1}$ & Rate & $\mathcal{N}_{h, \delta t}^{\infty}$ & Rate & $\min \widetilde{s}_{h, \delta t}$ \\
\hline 0.250 & $0.642 \mathrm{E}-04$ & - & $0.168 \mathrm{E}-04$ & - & $0.565 \mathrm{E}-03$ & - & $0.485 \mathrm{E}-05$ \\
0.125 & $0.345 \mathrm{E}-04$ & 1.074 & $0.879 \mathrm{E}-05$ & 0.939 & $0.241 \mathrm{E}-03$ & 1.231 & $0.300 \mathrm{E}-06$ \\
0.063 & $0.120 \mathrm{E}-04$ & 1.357 & $0.409 \mathrm{E}-05$ & 1.118 & $0.701 \mathrm{E}-04$ & 1.801 & $0.190 \mathrm{E}-07$ \\
0.031 & $0.360 \mathrm{E}-05$ & 1.698 & $0.133 \mathrm{E}-05$ & 1.579 & $0.185 \mathrm{E}-04$ & 1.876 & $0.115 \mathrm{E}-08$ \\
0.016 & $0.961 \mathrm{E}-06$ & 1.999 & $0.368 \mathrm{E}-06$ & 1.948 & $0.472 \mathrm{E}-05$ & 2.067 & $0.765 \mathrm{E}-09$ \\
\hline
\end{tabular}

Table 3: Numerical errors for the second example with $\lambda_{x}=\lambda_{y}=1$.

\begin{tabular}{|c||c|c|c|c|c|c||c|}
\hline $\mathrm{h}$ & $\mathcal{N}_{h, \delta t}^{2}$ & Rate & $\mathcal{N}_{h, \delta t}^{1}$ & Rate & $\mathcal{N}_{h, \delta t}^{\infty}$ & Rate & $\min \widetilde{s}_{h, \delta t}$ \\
\hline 0.250 & $0.111 \mathrm{E}+03$ & - & $0.402 \mathrm{E}+02$ & - & $0.498 \mathrm{E}+03$ & - & $0.157 \mathrm{E}-01$ \\
0.125 & $0.735 \mathrm{E}+02$ & 0.644 & $0.286 \mathrm{E}+02$ & 0.491 & $0.287 \mathrm{E}+03$ & 0.577 & $0.157 \mathrm{E}-01$ \\
0.063 & $0.459 \mathrm{E}+02$ & 0.688 & $0.180 \mathrm{E}+02$ & 0.674 & $0.190 \mathrm{E}+03$ & 0.603 & $0.156 \mathrm{E}-01$ \\
0.031 & $0.277 \mathrm{E}+02$ & 0.712 & $0.108 \mathrm{E}+02$ & 0.725 & $0.120 \mathrm{E}+03$ & 0.651 & $0.156 \mathrm{E}-01$ \\
0.016 & $0.163 \mathrm{E}+02$ & 0.805 & $0.622 \mathrm{E}+01$ & 0.832 & $0.755 \mathrm{E}+02$ & 0.698 & $0.156 \mathrm{E}-01$ \\
\hline
\end{tabular}

Table 4: Numerical errors for the second example with $\lambda_{x}=1$ and $\lambda_{y}=10^{-5}$.

\subsubsection{Example 3: Porous medium equation with a low regular solution}

This third test case continues with the porous medium equation where a particular emphasis is set on the low regularity in space of the analytical solution :

$$
s_{e}(x, y, t)=\max \left(2 \lambda_{x} t-x, 0\right), \quad \forall(x, y, t) \in \Omega \times\left(0, t_{f}\right) .
$$

We have utilized the isotropic permeability $\lambda_{x}=\lambda_{y}=1$ and the anisotropic one $\lambda_{x}=1$, $\lambda_{y}=100$ to test the efficiency of our discretization. Tables 5-6 exhibit the corresponding 
results. In the first table we observe that the convergence rate is strictly less than two which is not surprising since the exact solution does not belong to $H^{2}(\Omega)$ in space. In addition to this fact, the method becomes of an order approaching to 1 when $h$ decreases in the anisotropic case as highlighted in the second table. Negligible undershoots are also noted when the ratio $\lambda_{y} / \lambda_{x}$ increases.

\begin{tabular}{|c||c|c|c|c|c|c||c|}
\hline $\mathrm{h}$ & $\mathcal{N}_{h, \delta t}^{2}$ & Rate & $\mathcal{N}_{h, \delta t}^{1}$ & Rate & $\mathcal{N}_{h, \delta t}^{\infty}$ & Rate & $\min \widetilde{s}_{h, \delta t}$ \\
\hline 0.250 & 0.305 E-02 & - & 0.505 E-03 & - & 0.352 E-01 & - & 0 \\
0.125 & 0.122 E-02 & 1.326 & 0.186 E-03 & 1.439 & 0.184 E-01 & 0.940 & 0 \\
0.063 & 0.449 E-03 & 1.454 & 0.596 E-04 & 1.663 & 0.903 E-02 & 1.034 & 0 \\
0.031 & 0.163 E-03 & 1.430 & 0.181 E-04 & 1.681 & 0.446 E-02 & 0.994 & 0 \\
0.016 & 0.578 E-04 & 1.567 & 0.522 E-05 & 1.880 & 0.221 E-02 & 1.062 & 0 \\
\hline
\end{tabular}

Table 5: Numerical errors for the third example with $\lambda_{x}=\lambda_{y}=1$.

\begin{tabular}{|c||c|c|c|c|c|c||c|}
\hline $\mathrm{h}$ & $\mathcal{N}_{h, \delta t}^{2}$ & Rate & $\mathcal{N}_{h, \delta t}^{1}$ & Rate & $\mathcal{N}_{h, \delta t}^{\infty}$ & Rate & $\min \widetilde{s}_{h, \delta t}$ \\
\hline 0.250 & 0.136 E-01 & - & 0.318 E-02 & - & 0.109 E-00 & - & -0.444 E-21 \\
0.125 & 0.967 E-02 & 0.488 & 0.227 E-02 & 0.485 & 0.855 E-01 & 0.344 & -0.157 E-26 \\
0.063 & 0.686 E-02 & 0.501 & 0.148 E-02 & 0.627 & 0.681 E-01 & 0.332 & -0.151 E-33 \\
0.031 & 0.482 E-02 & 0.496 & 0.931 E-03 & 0.651 & 0.527 E-01 & 0.360 & -0.297 E-41 \\
0.016 & 0.332 E-02 & 0.562 & 0.568 E-03 & 0.746 & 0.407 E-01 & 0.393 & -0.795 E-25 \\
\hline
\end{tabular}

Table 6: Numerical errors for the third example with $\lambda_{x}=1$ and $\lambda_{y}=100$.

To conclude this subsection, the convergence speed obtained by our approach is visibly sensible to higher anisotropic fields. The same issue has been underlined in [8]. This is mainly due to the choice (6.5) and the degeneracy character of the model problem (6.1). We however conjecture that the method is at most of first order for any permeability tensor. We further stress that it provides no undershoots nor overshoots. Compared to [8] our scheme seems to behave better since the convergence rates are significantly enhanced. These latter results, especially the first order accuracy, are somehow satisfactory and turn out the scheme in question a robust compromise for the discretization of challenging complex problems. This motivates the application of our idea to the diphasic compressible model since the mobilities of both phases are often approximated by first order upwind schemes as investigated in many works $[4,6,7,29,32]$ and the references are therein.

\subsection{Numerical experiments for a diphasic compressible flow in anisotropic media}

The physical data used in these tests are being inspired by [32]. This experiment is termed as the five-spot problem in the literature. The goal is to simulate the secondary recovery of gas occurring in a $2 \mathrm{D}$ anisotropic reservoir with respect to the permeability tensor. The domain of computation representing the reservoir of interest is of length $L x=1 \mathrm{~m}$ and width $L y=1 \mathrm{~m}$. It is covered by 3584 triangles which makes 1857 dual control volumes as depicted in Figure 2.

We then consider the uniform porosity $\phi=0.206$. The phases of reference are gas as the nonwetting phase and water as the wetting one. This is not an exhaustive choice, one may take other options. We assume that the gas density obeys the well-known ideal gas law:

$$
\rho_{g}\left(p_{g}\right)=\rho_{g, r e f} \frac{p_{g}}{P_{g, r e f}},
$$




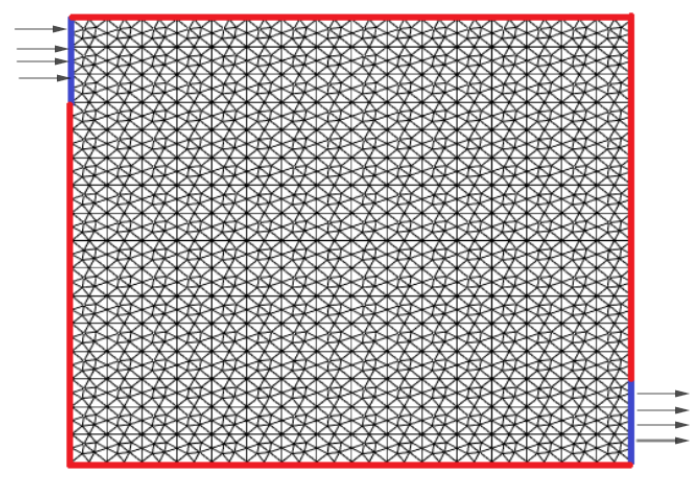

Figure 2: Primal mesh with 3584 triangles.

where we have fixed $\rho_{g, r e f}$ and $P_{g, r e f}$ to

$$
\rho_{g, r e f}=400 \mathrm{Kg} \cdot \mathrm{m}^{-3}, P_{g, r e f}=1.013 \times 10^{5} \mathrm{~Pa} .
$$

On the other hand, the water phase is weakly compressible with regards to the gas. Therefore, its density follows an exponential law with a small compressibility coefficient. Accordingly, we consider

$$
\rho_{w}\left(p_{w}\right)=\rho_{w, r e f}\left(1+c_{r e f}\left(p_{w}-P_{w, r e f}\right)\right),
$$

where we have set

$$
\rho_{w, r e f}=1000 \mathrm{Kg} \cdot \mathrm{m}^{-3}, c_{\text {ref }}=10^{-6} \mathrm{~Pa}^{-1}, P_{w, r e f}=1.013 \times 10^{5} \mathrm{~Pa} .
$$

The choice of both densities is mainly practical, especially for computing the analytical expression of the nonlinear formula (3.6) for the $\alpha$-phase density $\rho_{\alpha, K L}^{n+1}$ at the interface between two control volumes $A_{K}$ and $A_{L}$.

The relative permeabilities are squared functions $K_{r, \alpha}\left(s_{\alpha}\right)=s_{\alpha}^{2}$. The fluid viscosities are respectively given by $\mu_{g}=9 \times 10^{-5} \mathrm{~Pa} . s, \mu_{w}=10^{-3} \mathrm{~Pa}$.s. The capillary pressure is considered to be a linear function in terms of the gas saturation $P_{c}\left(s_{g}\right)=P_{\max } s_{g}$ with $P_{\max }=10^{5} \mathrm{~Pa}$.

The domain is initially occupied by gas with a uniform saturation i.e. $s_{g}^{0}=1$ where the gaseous phase is at the standard atmospheric pressure $P_{g}^{0}=1.013 \times 10^{5} \mathrm{~Pa}$. Under the pressure $P_{w}^{l}=4.6732 \times 10^{5} \mathrm{~Pa}$, only water is injected in the upper left corner of the domain $(x=0$, $0.8 \leq y \leq 1)$ with $s_{w}^{l}=1$. This pressure allows to displace the gas which flows freely outside the medium. The extraction zone is located at the lower right corner $(x=1,0 \leq y \leq 0.2)$ and is considered at the atmospheric pressure. The rest of the boundary is impermeable as shown on Figure 2. No source or sink terms are imposed, which means that $f^{P}=f^{I}=0$.

\subsubsection{Test case 1 : Isotropic permeability}

In this test we consider that the medium is isotropic where the permeability matrix is given by

$$
\Lambda(x, y)=10^{-10}\left[\begin{array}{cc}
0.15 & 0 \\
0 & 0.15
\end{array}\right] \quad\left[m^{2}\right] .
$$

This expression of $\Lambda$ means that the water fluid flows within the medium regardless the space directions. Figure 3 illustrates the behavior of water saturation and gas pressure at $t_{1}=10 \mathrm{~s}$, $t_{2}=30 \mathrm{~s}$ and $t_{2}=60 \mathrm{~s}$. The saturation exhibits a smooth front which is due to the capillary forces. Since there is no principal axis, the water displaces diagonally the gas towards the extraction zone. It is also observed that almost the whole amount of gas is recovered at the final time. The compressibility of both phases especially of gas prevents the latter to exit immediately the medium after the injection process. In other words, when water is injected it takes a little 
bit time for gas to be produced at the production region. We indicate that at every time the computed saturation remains bounded between its physical ranges 0 and 1 .

To get a better idea on the flow, Figure 4 shows separately a cross-section of the wetting phase saturation and gas pressure profiles at the point $(0,0.9)$. After $t=50 \mathrm{~s}$, the water front reaches the exit. Also, the domain becomes increasingly filled by water and emptied from gas. On the other hand, the gas pressure is higher near the injector and lower when the right boundary is approached. It seems to be regular owing to the parabolic kind of the system. Even if it is not included in our theoretical part, it is shown that $P_{g}$ is bounded with $1.013 \times 10^{5} \mathrm{~Pa} \leq P_{g} \leq 4.6732 \times 10^{5} \mathrm{~Pa}$.
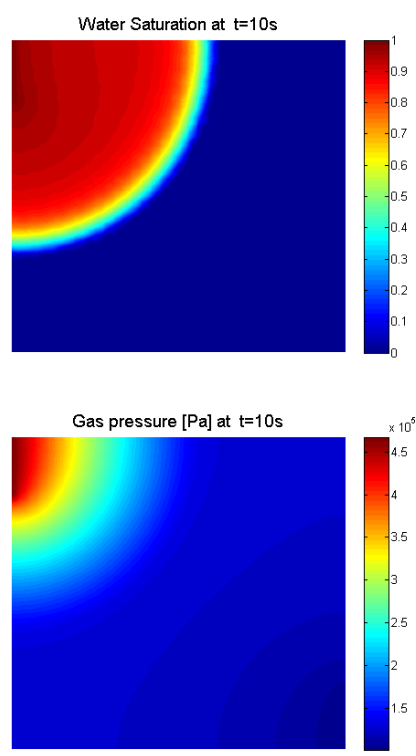

Water Saturation at $\mathrm{t}=30 \mathrm{~s}$

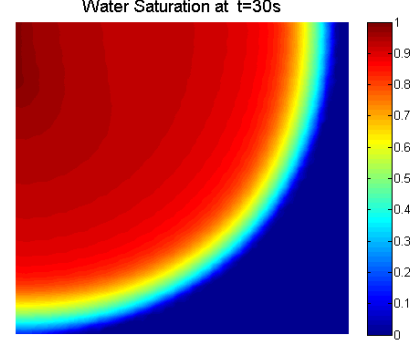

Gas pressure $[\mathrm{Pa}]$ at $\mathrm{t}=30 \mathrm{~s}$

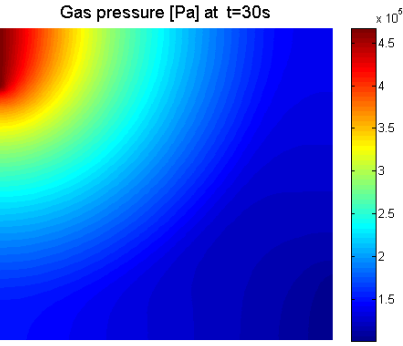

Water Saturation at $\mathrm{t}=60 \mathrm{~s}$

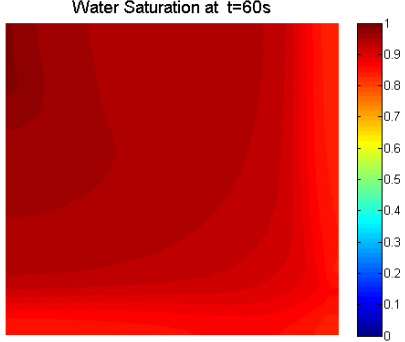

Gas pressure $[\mathrm{Pa}]$ at $\mathrm{t}=60 \mathrm{~s}$

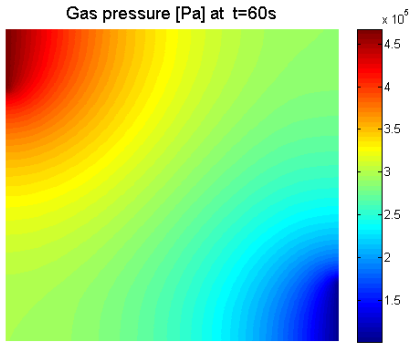

Figure 3: Test 1: Evolution of the water saturation (up) and the gas pressure (down) for simulation times $t_{1}=10 \mathrm{~s}, t_{2}=30 \mathrm{~s}$ and $t_{2}=60 \mathrm{~s}$ with the permeability choice (6.7).
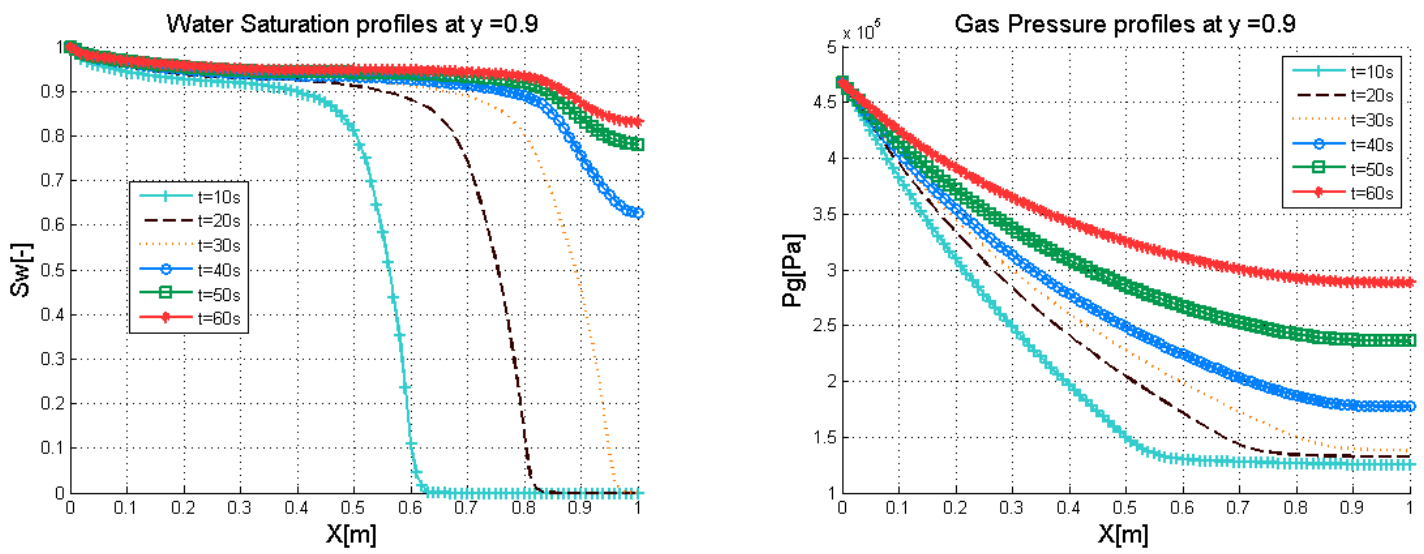

Figure 4: Test 1: Cross section of water saturation (left) and the gas pressure (right) at point $(0,0.9)$ for different times $t \in\{10 s, 20 s, 30 s, 40 s, 50 s, 60 s\}$. 


\subsubsection{Test case 2 : Homogeneous anisotropic permeability}

In this test, the permeability tensor is globally anisotropic. The flow action takes place in the direction obtained by rotating the vertical axis with the angle $\pi / 10$. Then

$$
\Lambda(x, y)=0.15 \times 10^{-10} \times R_{\theta_{0}} \times\left[\begin{array}{ll}
1 & 0 \\
0 & 5
\end{array}\right] \times R_{\theta_{0}}^{-1} \quad\left[m^{2}\right]
$$

where we have chosen $R_{\theta_{0}}$ for $\theta_{0}=\pi / 10$. This amounts to

$$
R_{\theta_{0}}=\left[\begin{array}{cc}
\cos \left(\theta_{0}\right) & -\sin \left(\theta_{0}\right) \\
\sin \left(\theta_{0}\right) & \cos \left(\theta_{0}\right)
\end{array}\right]=\left[\begin{array}{cc}
0.9511 & -0.3090 \\
0.3090 & 0.9511
\end{array}\right]
$$
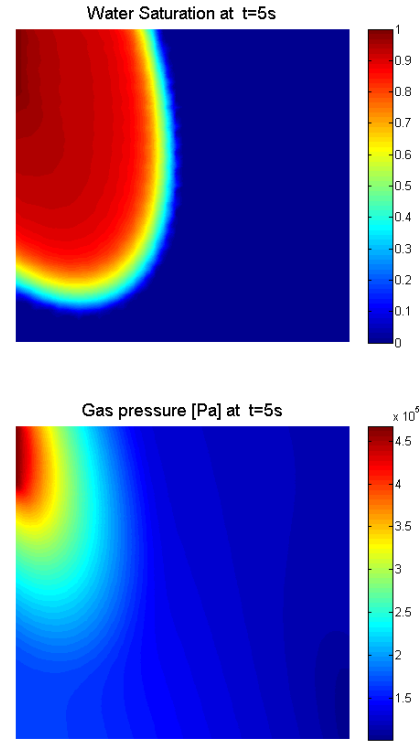

Water Saturation at $\mathrm{t}=20 \mathrm{~s}$

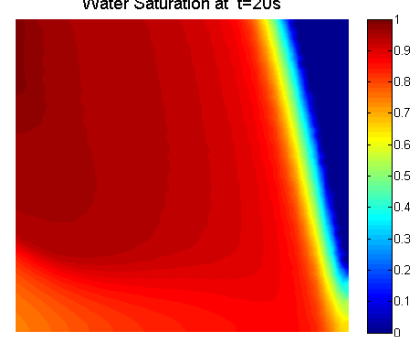

Gas pressure $[\mathrm{Pa}]$ at $\mathrm{t}=20 \mathrm{~s}$

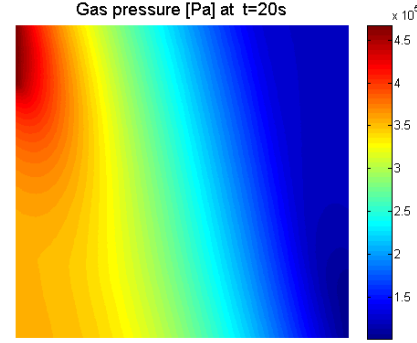

Water Saturation at $\mathrm{t}=60 \mathrm{~s}$

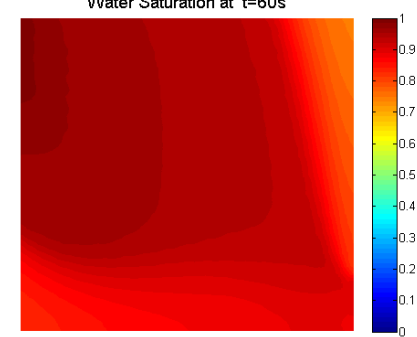

Gas pressure $[\mathrm{Pa}]$ at $\mathrm{t}=60 \mathrm{~s}$

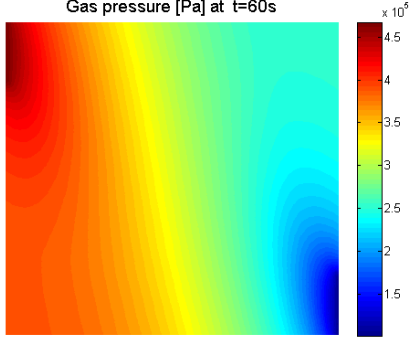

Figure 5: Test 2: Evolution of the water saturation (up) and the gas pressure (down) for simulation times $t_{1}=5 s, t_{2}=20 s$ and $t_{2}=60 s$ with the permeability choice (6.8).
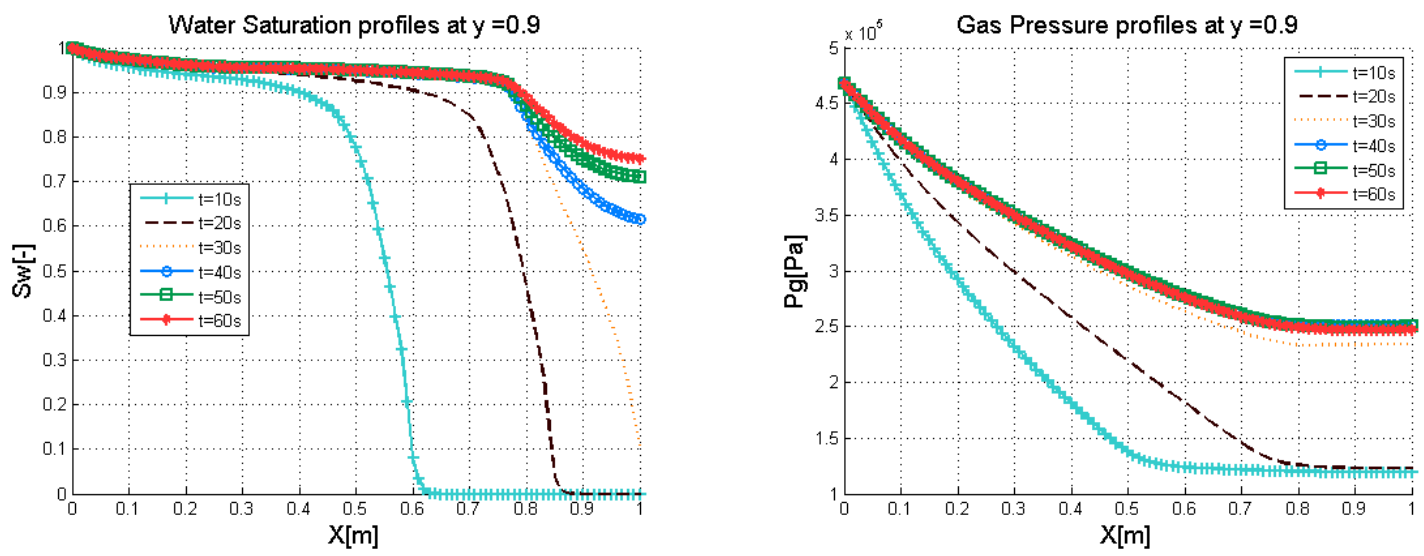

Figure 6: Test 2: Cross section of water saturation (left) and the gas pressure (right) at point $(0,0.9)$ for different times $t \in\{10 s, 20 s, 30 s, 40 s, 50 s, 60 s\}$. 
Figure 5 depicts the water saturation and gas pressure subject to capillary, compressibility and anisotropy constraints at different instants. However, our focus is set on the impact of the latter kinds of effects. It is seen that the injection of water is keeping the movement of the gas from the left side towards the right side. This is of course ensured by the high pressure which is maintained in the $y$-direction rotated by $\pi / 10$ in the negative sense. Compared to Test 1 , the $y$-eigenvalue is much bigger in this test, therefore the pores are relatively large in this direction. Consequently, more than the half amount of gas is recuperated in a short time as we observe on the figure. It is also noticed that after $t=20 \mathrm{~s}$ water becomes to leave the domain where a considerable quantity of gas is remained therein. We emphasize that the calculated saturation is always delimited by its boundary values 0 and 1 which confirms the theoretical result established in Lemma 4.1 .

The cross-section of the water profile presented in Figure 6 at the point $(0,0.9)$ shows that the flow with respect to the $x$-axis is slightly similar (up to a rotation) to the first test case. The gas pressure varies slowly at the begging of the recovery operation and achieves somehow a threshold when the medium becomes to be saturated by water.

\subsubsection{Test case 3 : Heterogeneous rotating anisotropic permeability}

This test ensures the ability of our method to simulate the considered compressible two-phase flow through a heterogeneous medium in terms of its permeability. The expression of this property is taken from $[28,25]$ :

$$
\Lambda(x, y)=\frac{0.15 \times 10^{-10}}{x^{2}+y^{2}}\left[\begin{array}{cc}
0.1 x^{2}+y^{2} & 0.9 x y \\
0.9 x y & x^{2}+0.1 y^{2}
\end{array}\right] \quad\left[m^{2}\right], \quad \forall(x, y) \in \Omega .
$$

Here the flow of both fluids depends on the position since the permeability varies in space. However, we continue by injecting water as in the previous tests. Figure 7 displays the behavior of water in the medium. It is recorded that the wetting phase displaces faster horizontally. Obviously, this fact is caused by the heterogeneity of the medium and therefore the location of the injector. Indeed, at this spot, the $x$-variable is closer to 0 while the $y$-variable is around 1 . Consequently, the flow velocity is important around this area as the pressure sub-figures expose. As the $x$-variable increases we see that the movement is relatively slowed which permits the entry of water in the diagonal of the domain. Here again the saturation presents no oscillation and is bounded between its natural limits 0 and 1 . The same remark is made on the pressure.

Once more, the cross-section of the water saturation and gas pressure profiles is given in Figure 8 at $(0,0.9)$. It is indicated that the process is taking more and more time to move all the gas outside of the medium. This is natural since the pores are too tiny. We also remark that the pressure changes its convexity shape.

\subsubsection{Test case 4: Heterogeneous rotating anisotropic permeability with a nondegenerate bounded gas density}

In this last test case we place our attention on the flow where the density is satisfying Assumption $\left(\mathbf{A}_{5}\right)$. This means that the gas density should be uniformly bounded, especially away from 0 . In this case, the problem is nondegenerate with respect to the gas pressure. To achieve this situation, we keep all the data of the preceding Test case 3 except the gas density whose expression is now given by :

$$
\rho_{g}\left(p_{g}\right)=\rho_{g, r e f}\left(1+\gamma_{g}\left(p_{g}-P_{g, r e f}\right)\right)
$$

where $\gamma$ is chosen as $\gamma=10^{-4}$. It accounts for a compressibility factor. As previously, injecting water on a part of the boundary with on overpressure allows its spreading within the medium. We observe that the flow path obeys the preferential axes determined by the anisotropy of the permeability. As expected, one observes on Figure 9 that the water takes less time compared to 

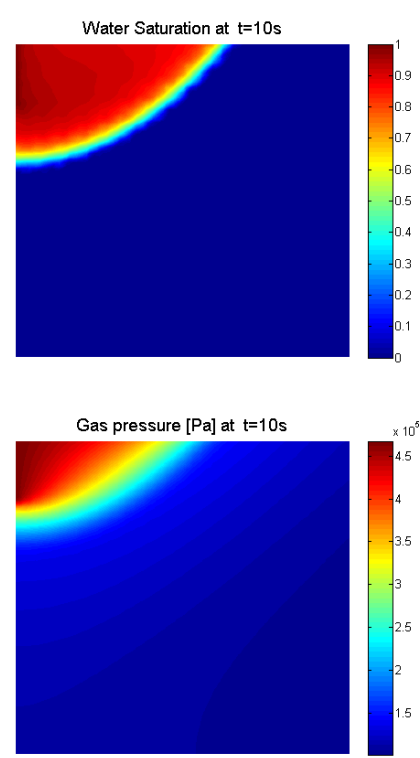
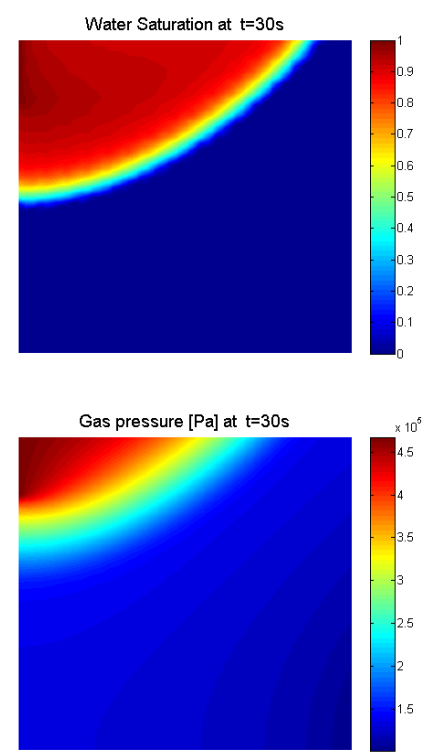
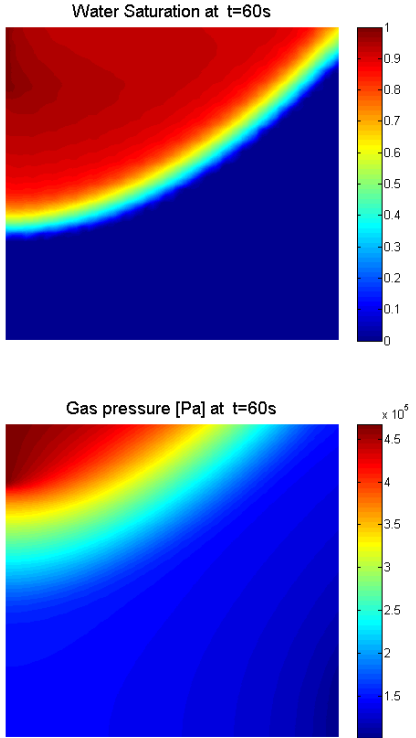

Figure 7: Test 3: Evolution of the water saturation (up) and the gas pressure (down) for simulation times $t_{1}=10 \mathrm{~s}, t_{2}=30 \mathrm{~s}$ and $t_{2}=60 \mathrm{~s}$ with the permeability choice (6.9).
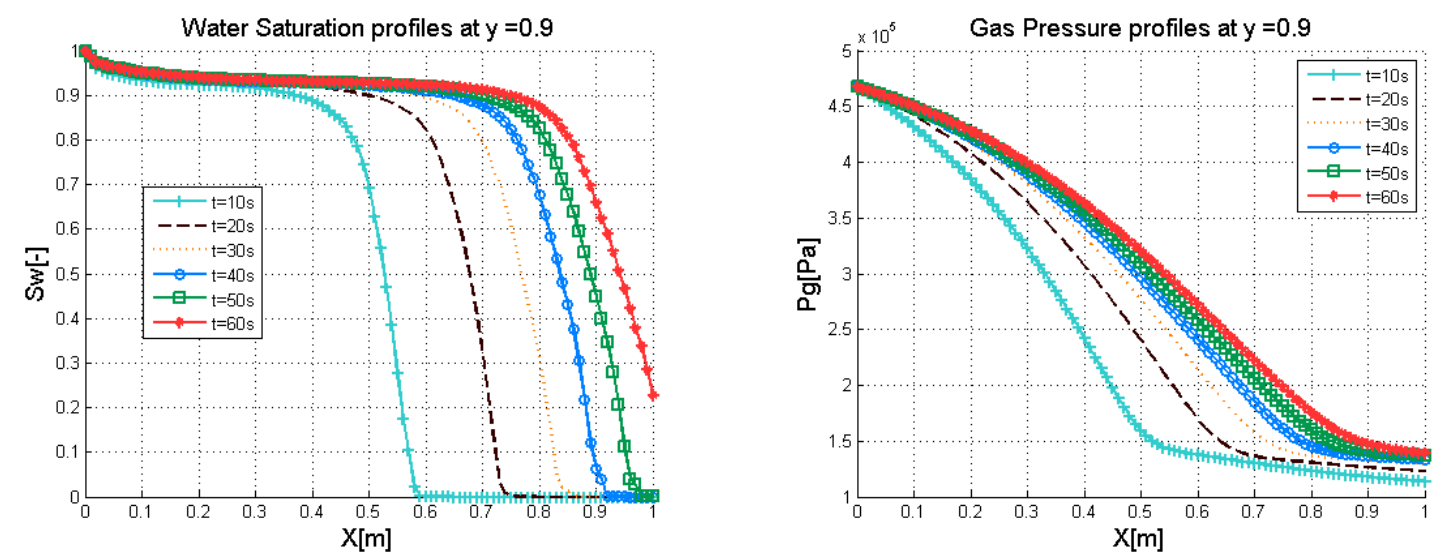

Figure 8: Test 3: Cross section of water saturation (left) and the gas pressure (right) at point $(0,0.9)$ for different times $t \in\{10 s, 20 s, 30 s, 40 s, 50 s, 60 s\}$.

Test case 3 to invade the reservoir. This goes back to the additional transport term coming from the nondegeneracy effects of the gas density. A similar remark is clearly seen on the evolution of the gas pressure. The water saturation remains always between its natural ranges 0 and 1 . To confirm these facts from a close viewpoint, we refer to the cross-section of the water saturation and gas pressure profiles at $(0,0.9)$ given in Figure 10. As a consequence of the nondegeneracy influence of the density function, a faster displacement of water is observed compared to Test case 3 and a rapidly dispersive behavior of the gas pressure is noticed.

To summary, we presented the qualitative behavior of water saturation and the gas pressure as a discrete solution to the numerical scheme (3.1)-(3.5). In the all aforementioned tests we have focused our attention on the displacement of phases through the porous medium in the presence of anisotropy of the permeability tensor. We noticed that the flow paths follow naturally the principal axis, which matches with the real case. Moreover, numerical results showed that the velocity of the flow is strongly depending on the choice of the phase density. We have also seen 

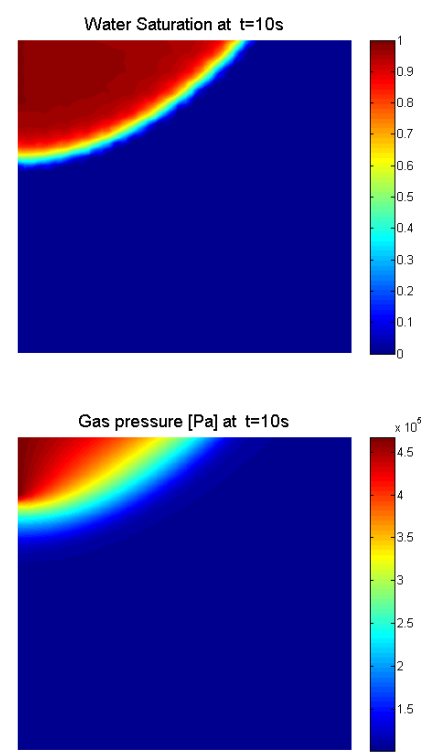

Water Saturation at $\mathrm{t}=30 \mathrm{~s}$

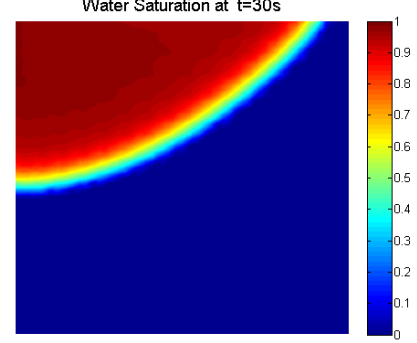

Gas pressure $[\mathrm{Pa}]$ at $\mathrm{t}=30 \mathrm{~s}$

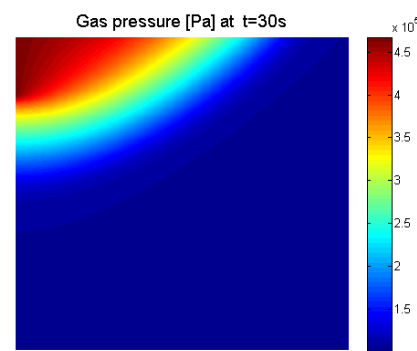

Water Saturation at $\mathrm{t}=60 \mathrm{~s}$

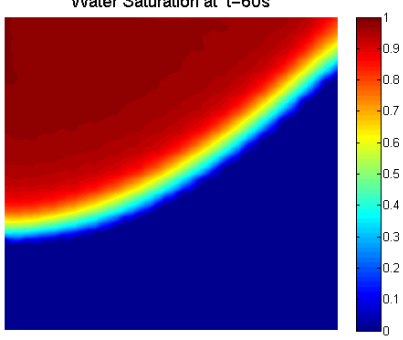

Gas pressure $[\mathrm{Pa}]$ at $\mathrm{t}=60 \mathrm{~s}$

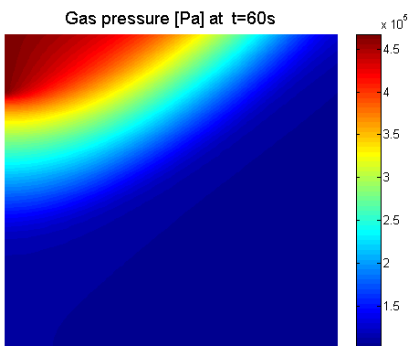

Figure 9: Test 4: Evolution of the water saturation (up) and the gas pressure (down) for simulation times $t_{1}=10 \mathrm{~s}, t_{2}=30 \mathrm{~s}$ and $t_{2}=60 \mathrm{~s}$ with the permeability choice (6.9).
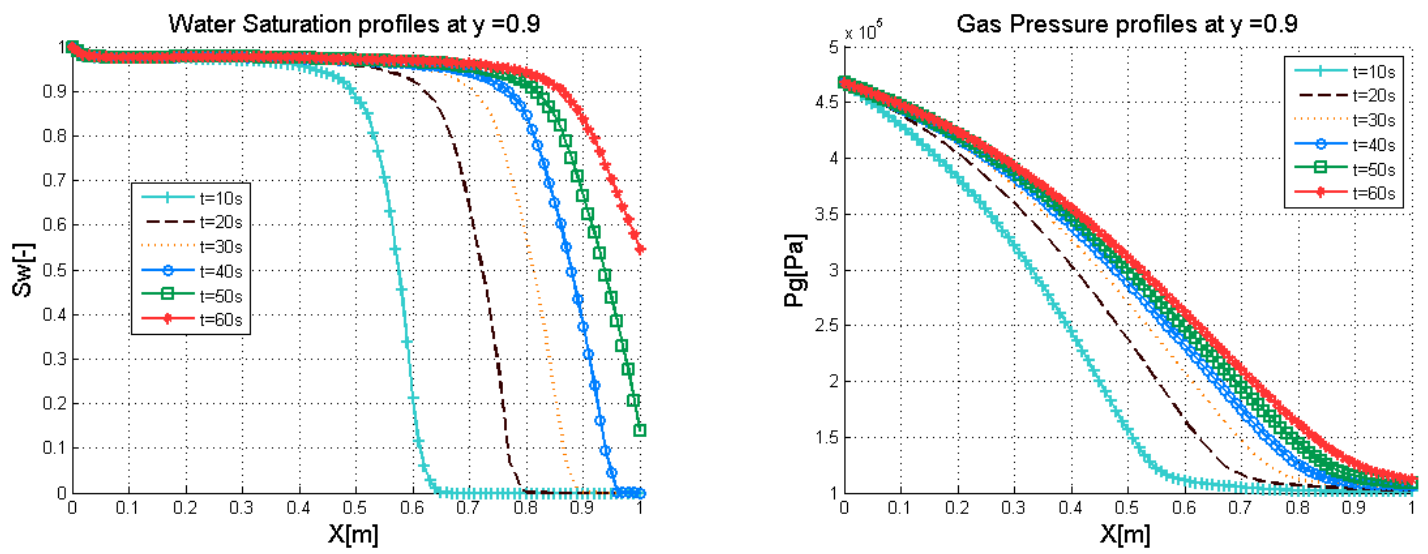

Figure 10: Test 4: Cross section of water saturation (left) and the gas pressure (right) at point $(0,0.9)$ for different times $t \in\{10 s, 20 s, 30 s, 40 s, 50 s, 60 s\}$.

that the proposed methodology respects the discrete maximum principle. On the other hand, this point has a theoretical target allowing to get the main ingredients, namely the energy and compactness estimates, for the convergence analysis of the scheme. It further provides a crucial physical information on the solution.

\section{A Appendix : On the positivity of the sub-upwinding scheme for a nonlinear diffusion problem}

In this appendix we show that the discretization (6.3)-(6.5) for the nonlinear diffusion problem (6.1) preserves a nonnegative lower bound on the solution. 
Lemma A.1. The numerical scheme (6.3)-(6.5) is positivity-preserving in the sense that

$$
\left(s_{K}^{0} \geq 0, \quad \forall K \in \mathcal{V}\right) \text { implies }\left(s_{K}^{n+1} \geq 0, \quad \forall K \in \mathcal{V}, n \in \llbracket 0, N-1 \rrbracket\right) .
$$

Proof. To prove the above statement we resort to the induction. We select $n \in \llbracket 0, N-1 \rrbracket$. Let us assume that $s_{K}^{l} \geq 0$ for every $l \leq n$. We now check that $s_{K}^{n+1} \geq 0$ for all $K \in \mathcal{V}$. We still denote $K$ the vertex of $\mathcal{V}$ such that $s_{K}^{n+1}=\min _{L \in \mathcal{V}} s_{L}^{n+1}$. The case $s_{K}^{n+1} \geq 0$ is trivial. We therefore assume that $s_{K}^{n+1}<0$. We next multiply the line of the system (6.5) associated to $K$ by $\left(s_{K}^{n+1}\right)^{-}=-\min \left(s_{K}^{n+1}, 0\right)$ to get

$$
-\left|\left(s_{K}^{n+1}\right)^{-}\right|^{2}-s_{K}^{n}\left(s_{K}^{n+1}\right)^{-}-\frac{\delta t}{\left|A_{K}\right|} \sum_{T \in \mathcal{K}_{\mathcal{T}_{h}}} \sum_{L \in \mathcal{V}_{K T}} \Lambda_{K L}^{T} a_{K L}^{T}\left(f\left(s_{L}^{n+1}\right)-f\left(s_{K}^{n+1}\right)\right)\left(s_{K}^{n+1}\right)^{-}=0 .
$$

Using the fact that the function $a(s)$ is extended by 0 for all $]-\infty, 0\left[\right.$ and the definition of $a_{K L}^{T}$ given in (6.5), one obtains $a_{K L}^{T}\left(s_{K}^{n+1}\right)^{-}=0$ in the case where $\Lambda_{K L}^{T}<0$. Consequently

$$
\underbrace{-\left|\left(s_{K}^{n+1}\right)^{-}\right|^{2}}_{X} \underbrace{-s_{K}^{n}\left(s_{K}^{n+1}\right)^{-}}_{Y}-\underbrace{-\frac{\delta t}{\left|A_{K}\right|} \sum_{T \in \mathcal{K}_{T_{h}}} \sum_{L \in \mathcal{V}_{K T}}\left(\Lambda_{K L}^{T}\right)^{+} a_{K L}^{T}\left(f\left(s_{L}^{n+1}\right)-f\left(s_{K}^{n+1}\right)\right)\left(s_{K}^{n+1}\right)^{-}}_{Z}=0 .
$$

On the other hand, the quantity $Y$ is nonpositive thanks to the induction assumption $s_{K}^{n}$. Also, one has $Z \leq 0$ since the function $f$ is nondecreasing. We then deduce

$$
\left(s_{K}^{n+1}\right)^{-}=0,
$$

which yields a contradiction with the assumption $\left(s_{K}^{n+1}\right)^{-}<0$. Therefore $s_{K}^{n+1} \geq 0, \forall K \in \mathcal{V}$. The proof is concluded.

\section{References}

[1] M. Afif and B. Amaziane. Convergence of finite volume schemes for a degenerate convection-diffusion equation arising in flow in porous media. Computer methods in applied mechanics and engineering, 191(46):5265-5286, 2002.

[2] B. Amaziane, M. Jurak, and A. Ž. Keko. An existence result for a coupled system modeling a fully equivalent global pressure formulation for immiscible compressible two-phase flow in porous media. Journal of Differential Equations, 250(3):1685-1718, 2011.

[3] B. Andreianov, M. Bendahmane, and R. Ruiz-Baier. Analysis of a finite volume method for a cross-diffusion model in population dynamics. Mathematical Models and Methods in Applied Sciences, 21(2):307-344, 2011.

[4] B. Andreianov, R. Eymard, M. Ghilani, and N. Marhraoui. Finite volume approximation of degenerate two-phase flow model with unlimited air mobility. Numerical Methods for Partial Differential Equations, 29(2):441-474, 2013.

[5] J. Bear and Y. Bachmat. Introduction to Modeling of Transport Phenomena in Porous Media, volume 4 of Theory and Applications of Transport in Porous Media. Kluwer Academic Publishers, Dordrecht, Holland, 1990.

[6] M. Bendahmane, Z. Khalil, and M. Saad. Convergence of a finite volume scheme for gaswater flow in a multi-dimensional porous medium. Mathematical Models and Methods in Applied Sciences, 24(1):145-185, 2014. 
[7] K. Brenner and R. Masson. Convergence of a vertex centred discretization of two-phase Darcy flows on general meshes. International Journal on Finite Volumes, 10:1-37, 2013.

[8] C. Cancès and C. Guichard. Convergence of a nonlinear entropy diminishing control volume finite element scheme for solving anisotropic degenerate parabolic equations. Mathematics of Computation, 85(298):549-580, 2016.

[9] C. Cancès and C. Guichard. Numerical analysis of a robust free energy diminishing finite volume scheme for parabolic equations with gradient structure. Foundations of Computational Mathematics, 17(6):1525-1584, 2017.

[10] C. Cancès, M. Ibrahim, and M. Saad. Positive nonlinear CVFE scheme for degenerate anisotropic Keller-Segel system. SMAI Journal of Computational Mathematics, 3:1-28, 2017.

[11] G. Chavent and J. Jaffré. Mathematical models and finite elements for reservoir simulation: single phase, multiphase and multicomponent flows through porous media, volume 17. North-Holland, Amsterdam, Stud. Math. Appl. edition, 1986.

[12] Z. Chen and R. E. Ewing. Fully discrete finite element analysis of multiphase flow in groundwater hydrology. SIAM Journal on numerical analysis, 34(6):2228-2253, 1997.

[13] Z. Chen, G. Huan, and Y. Ma. Computational methods for multiphase flows in porous media, volume 2. SIAM, Pennsylvania, USA, Philadephia edition, 2006.

[14] J. Droniou. Finite volume schemes for diffusion equations: introduction to and review of modern methods. Mathematical Models and Methods in Applied Sciences, 24(8):1575-1619, 2014.

[15] Y. Epshteyn and B. Rivière. Fully implicit discontinuous finite element methods for twophase flow. Applied Numerical Mathematics, 57(4):383-401, 2007.

[16] A. Ern and J.-L. Guermond. Theory and practice of finite elements, volume 159. Springer Science \& Business Media, 2013.

[17] L. C. Evans. Partial differential equations. 2nd edn. Amer. Math. Soc., 2010.

[18] R. Eymard and T. Gallouët. Convergence d'un schéma de type éléments finis-volumes finis pour un système formé d'une équation elliptique et d'une équation hyperbolique. ESAIM: Mathematical Modelling and Numerical Analysis, 27(7):843-861, 1993.

[19] R. Eymard, T. Gallouët, C. Guichard, R. Herbin, and R. Masson. TP or not TP, that is the question. Computational Geosciences, 18(3-4):285-296, 2014.

[20] R. Eymard, T. Gallouët, and R. Herbin. Finite volume methods. In Handbook of Numerical Analysis, volume 7, pages 713-1018. Elsevier, 2000.

[21] R. Eymard, R. Herbin, and A. Michel. Mathematical study of a petroleum-engineering scheme. ESAIM: Mathematical Modelling and Numerical Analysis, 37(6):937-972, 2003.

[22] C. Galusinski and M. Saad. A nonlinear degenerate system modelling water-gas flows in porous media. Discrete and Continuous Dynamical Systems Series B, 9(2):281, 2008.

[23] M. Ghilani, E. H. Quenjel, and M. Saad. Positive control volume finite element scheme for a degenerate compressible two-phase flow in anisotropic porous media. Computational Geosciences, 23(1):55-79, 2019.

[24] R. Helmig. Multiphase flow and transport processes in the subsurface: a contribution to the modeling of hydrosystems. Springer-Verlag, 1997. 
[25] R. Herbin and F. Hubert. Benchmark on discretization schemes for anisotropic diffusion problems on general grids. In R. Eymard and J.-M. Herard, editors, Finite Volumes for Complex Applications V, pages 659-692. Wiley, 2008.

[26] R. Huber and R. Helmig. Node-centered finite volume discretizations for the numerical simulation of multiphase flow in heterogeneous porous media. Computational Geosciences, $4(2): 141-164,2000$.

[27] Z. Khalil and M. Saad. Solutions to a model for compressible immiscible two phase flow in porous media. Electronic Journal of Differential Equations, 122:1-33, 2010.

[28] C. Le Potier. Schéma volumes finis pour des opérateurs de diffusion fortement anisotropes sur des maillages non structurés. Comptes Rendus Mathematique, 340(12):921-926, 2005.

[29] A. Michel. A finite volume scheme for two-phase immiscible flow in porous media. SIAM Journal on Numerical Analysis, 41(4):1301-1317, 2003.

[30] I. Mozolevski and L. Schuh. Numerical simulation of two-phase immiscible incompressible flows in heterogeneous porous media with capillary barriers. Journal of Computational and Applied Mathematics, 242:12-27, 2013.

[31] M. Ohlberger. Convergence of a mixed finite element: Finite volume method for the two phase flow in porous media. East West Journal of Numerical Mathematics, 5:183-210, 1997.

[32] B. Saad and M. Saad. Study of full implicit petroleum engineering finite-volume scheme for compressible two-phase flow in porous media. SIAM Journal on Numerical Analysis, 51(1):716-741, 2013.

[33] B. Saad and M. Saad. A combined finite volume-nonconforming finite element scheme for compressible two phase flow in porous media. Numerische Mathematik, 129(4):691-722, 2015.

[34] I. Yotov. A mixed finite element discretization on non-matching multiblock grids for a degenerate parabolic equation arising in porous media flow. East West Journal of Numerical Mathematics, 5:211-230, 1997. 